\title{
Consensus Algorithms for Biased Labeling in Crowdsourcing
}

\author{
Jing Zhang a, b, Victor S. Sheng ${ }^{c, d}$, Qianmu $\mathrm{Li}^{\mathrm{a}}{ }^{\mathrm{a}, \mathrm{b}}$, Jian $\mathrm{Wu}^{\mathrm{e}}$, Xindong $\mathrm{Wu}^{\mathrm{f}}$ \\ a. School of Computer Science and Engineering, Nanjing University of Science and Technology, \\ Nanjing 210094, China \\ b. Key Laboratory of Image and Video Understanding for Social Safety (Nanjing University of Science \\ and Technology), Nanjing, 210094, China \\ c. Department of Computer Science, University of Central Arkansas, Conway, AR 72035, U.S. \\ d. Jiangsu Engineering Center of Network Monitoring, Nanjing University of Information Science and \\ Technology, Nanjing 210044, China \\ e. Soochow University, Suzhou 215006, China \\ f. School of Computing and Informatics, University of Louisiana at Lafayette, LA 70504, U.S.
}

\begin{abstract}
Although it has become an accepted lay view that when labeling objects through crowdsourcing systems, non-expert annotators often exhibit biases, this argument lacks sufficient evidential observation and systematic empirical study. This paper initially analyzes eight real-world datasets from different domains whose class labels were collected from crowdsourcing systems. Our analyses show that biased labeling is a systematic tendency for binary categorization; in other words, for a large number of annotators, their labeling qualities on the negative class (supposed to be the majority) are significantly greater than are those on the positive class (minority). Therefore, the paper empirically studies the performance of four existing EM-based consensus algorithms, DS, GLAD, RY, and ZenCrowd, on these datasets. Our investigation shows that all of these state-of-the-art algorithms ignore the potential bias characteristics of datasets and perform badly although they model the complexity of the systems. To address the issue of handling biased labeling, the paper further proposes a novel consensus algorithm, namely adaptive weighted majority voting (AWMV), based on the statistical difference between the labeling qualities of the two classes. AWMV utilizes the frequency of positive labels in the multiple noisy label set of each example to obtain a bias rate and then assigns weights derived from the bias rate to negative and positive labels. Comparison results among the five consensus algorithms (AWMV and the four existing) show that the proposed AWMV algorithm has the best overall performance. Finally, this paper notes some potential related topics for future study.

Keywords: labeling bias, crowdsourcing, EM algorithm, consensus, weighted majority voting
\end{abstract}

\section{Introduction}

Crowdsourcing [14] systems provide convenient platforms to collect human intelligence for a variety of problems that computers are currently unable to handle, from micro-tasks, such as movie ranking [38], polarity annotation[3], and image tagging [36], to gathering global talents involving a large research innovation [8]. Amazon Mechanical Turk (MTurk) is one of the most successful systems, providing a marketplace for individuals or businesses to conduct their Human Intelligence Task (HIT) 
transactions. Text annotating, image tagging, and website ranking are being completed online using crowdsourcing systems, saving significant time and labor cost compared with a traditional operational mode - hiring professionals to perform the tasks.

However, crowdsourcing faces two main challenges when class labels of objects are provided by nonprofessional (crowd) annotators recruited from the Internet: (1) uneven qualities of their labeling behaviors, and (2) lack of prior knowledge. To improve qualities of the collected data for subsequent model learning, it is common to obtain multiple labels for one object from different annotators. Then, an integrated label will be inferred from these repeated labels using a consensus algorithm. Because the true label of the object is unknown, the goal of the consensus algorithm is to infer the ground truth from the wisdom of the crowd (annotations). Note that in the ground truth inference, no prior knowledge can be utilized to help the algorithm make decisions. On the one hand, we do not have any historical information to analyze the labeling qualities of these annotators, due to causes that include the open nature of the online marketplace, privacy protection [9] [31], and the lack of a mature quality control mechanism. On the other hand, thoroughly analyzing the qualities of these annotators by humans costs too much work and intelligence. Therefore, the consensus algorithm must be agnostic and not use any prior knowledge such as the prior qualities of annotators, difficulties of the objects, or the underlying class distribution of objects.

The simplest efficient consensus algorithm is majority voting (MV), which results in a high quality of the integrated labels of instances, as long as the accuracies of the noisy labels provided by annotators lie in a certain range [15] [17] [32]. Unfortunately, real-world environments are so complicated that MV does not always work well, particularly under situations in which biases exist in the labeling behaviors of annotators. With binary categorization for example, biased annotators can provide one type of label at a very high probability. When one opinion completely overwhelms another one, MV fails. Note that the bias is different from the individual error of an annotator. It is a systematic tendency caused by causes such as lack of expertise, inconsistent judgment criteria, and interests and preferences of annotators. Annotators might carefully complete the task but still have biases. For example, when judging whether a website contains adult content, to protect their children to the greatest extent, most parents would rather treat some regular websites as being harmful to the youth than vice versa. Similarly, in the task of describing the sentiments of news headlines, ordinary people usually fail to perceive the sentiment of a news headline. On the one hand, they have insufficient expertise to understand the potential meaning that a headline tries to convey. On the other hand, news headlines often use impartial-sounding language. It is no exaggeration to say that biased labeling is an innate attribute of crowdsourcing.

Several more sophisticated agnostic consensus algorithms such as GLAD [42], RY [30], and ZenCrowd [6] have been proposed in recent years. Some of them [30] [41] are conscious of the existence of bias, but their performance on the biased wisdom of crowdsourcing has not been well investigated. This paper systematically studies the biased labeling issue and tries to answer the following questions. (1) What is bias? How does one characterize bias? (2) Does it really exist in real-world crowdsourcing systems? Are the deviation directions of most annotators in the system consistent? (3) How do the state-of-the-art consensus algorithms perform on these biased data? (4) How does the bias affect subsequent model learning procedures?

The contributions of this paper are four fold. (1) This is the first paper to analyze and reveal the widespread potential biased labeling phenomenon in real-world datasets. By detailed analyses of eight 
real-world datasets, the hypothesis of biased labeling is strongly supported. (2) We propose a novel consensus algorithm, namely adaptive weighted majority voting (AWMV), to address the biased labeling issue. We not only show that AWMV outperforms the other four state-of-the-art EM-based consensus algorithms but also note the reasons why some EM-based algorithms do not work under biased labeling scenarios. (3) This paper is the first to highlight the capability of correctly inferring the minority, which is ignored by the state-of-the-art consensus algorithms and is important for subsequent model learning. (4) Potential research issues related to the biased wisdom are listed at the end of the paper to attract more attention and contributions.

The remainder of this paper is organized as follows. Section 2 briefly reviews the literature related to the paper. In Section 3, the formal definition of the problem is presented, followed by four EM-based algorithms. Section 4 describes the proposed AWMV algorithm in detail. In Section 5, a series of experiments are conducted and their results are thoroughly analyzed. Section 6 concludes the paper and provides open topics for future study.

\section{Related Work}

About real-world datasets and the biased labeling issue. To investigate whether crowdsourcing can be actually used in natural language processing tasks, Snow et al. posted five linguistic annotation problems on Amazon Mechanical Turk, collected a set of noisy labels for each problem and shared them through open access [35]. Thereafter, many recent research articles were published, providing free access to their real-world datasets [4] [16] [19] [41] [42]. In addition to these articles, some academic conferences began to introduce crowdsourcing tracks, with the related real-world crowdsourced datasets published after the sessions, such as TREC 2010-2013, HCOMP 2013 and MediaEval 2013. We only include the datasets with ground truth (gold) labels for evaluation. Errors in ground truth labels can affect the evaluation of algorithms [2] [21]. However, we ignore this problem in this paper. Some previous studies mentioned the biased labeling issue [16] [26] [30] [46]. Biased labeling will result in imbalanced learning if consensus algorithms cannot handle this issue well [43] [44]. Imbalanced class distributions together with label noise bring considerable difficulties to training a good model [7] [29]. However, none of the above studies provides deep analysis of real-world biased datasets or evaluates the approaches of these datasets.

About unsupervised consensus algorithms. Because of lack of prior knowledge, such as expertise of annotators, the level of bias, the underlying class distribution and the ground truth of unlabeled samples, an unsupervised consensus algorithm often plays an important role in crowdsourcing research. The straightforward consensus algorithm, MV, has been thoroughly studied [15] [32]. Besides MV, many consensus algorithms utilize the inference of a useful probabilistic model for estimating latent variables [1] [39]. The classic approach, DS [5] proposed by Dawid and Skene, models a confusion matrix of each annotator and the class prior and uses an EM procedure to infer the estimated labels of examples. Following these ideas, Smyth et al. inferred the ground truth of subjective labels on Venus images by using almost the same method [34]. Whitehill et al. proposed the GLAD algorithm, which models both the levels of expertise and the difficulties of examples, treating the probability of an object being positive as a latent variable [42]. Raykar et al. proposed a Bayesian approach RY to add specific priors for each class. In their approach, annotators have biases toward the positive class and toward the negative class, modeled by the two parameters sensitivity and specificity [30]. The two parameters correspond to the true positive rate and the true negative rate in the confusion matrix, respectively. Demartini, Difallah and Cudré-Mauroux proposed ZenCrowd, which only uses one parameter to model 
the reliability of each annotator and infers the true labels of examples [6]. It is slightly more complex than MV but simpler than DS and RY.

All of these EM-based algorithms simultaneously optimize the parameters of the model and estimate the true labels. Although EM is a general algorithm for inferring latent variables [24], it still suffers from the weakness of being trapped in a local optimum. There are also other non-EM-based consensus algorithms [18] [22] [40]. Welinder et al. [41] incorporated competence, expertise, and the difficulties of instances together to form a weight vector for each annotator. The probability of the ground truth is estimated using MAP estimation. Karger et al. [20] proposed a belief propagation model based on a low-rank matrix for label integration. Because the EM algorithm [27] is a general solution for unsupervised scenarios, it remains a mainstream fundamental approach for mining the wisdom of the crowds [23]. However, we argue that EM-based algorithms cannot handle biased labeling well by sufficient investigation of their performance on real-world datasets with bias. To solve the problem, we propose a non-EM-based algorithm AWMV in this paper. Weighted voting, derived directly from MV, shows its advantages in decision making with imbalance [17] [45].

About the comparison study of the consensus algorithms. In previous original studies of DS, GLAD, RY, and ZenCrowd, there is no research comparing these algorithms comprehensively on open accessible real-world datasets. Sheshadri and Lease recently constructed a benchmark platform for crowd consensus algorithms [33]. In their work, several open accessible datasets are integrated, and several algorithms implemented in Java are available. The platform also provides the comparison results of these algorithms on different datasets. However, it does not provide a detailed explanation and does not discuss the characteristics of the datasets. Some of the biased datasets studied in this paper are from [33]. Nguyen et al. presented a benchmarking framework using only synthetic data [28]. To the best of our knowledge, this study is the first to examine the performance of these algorithms on biased real-world datasets and to attempt to explain the corresponding latent reasons of each algorithm.

\section{Four EM-Based Consensus Algorithms}

In this section, we briefly review four existing EM-based consensus algorithms, DS, GLAD, RY and ZenCrowd. Before reviewing them, we initially introduce the problem definition of a consensus algorithm and notations used in this paper.

\subsection{Problem Definition of a Consensus Algorithm}

In a crowdsourcing system, a set of examples is denoted by $E=\left\{e_{i}\right\}_{i=1}^{I}$, where $e_{i}=<\boldsymbol{x}_{i}, y_{i}>, \boldsymbol{x}_{i}$ is the feature portion and $y_{i}$ is the true label of the example. A set of annotators is denoted by $U=\left\{u_{j}\right\}_{j=1}^{J}$. Each label belongs to a set of classes $C=\left\{c_{k}\right\}_{k=1}^{K}$. For convenience, we can use its index as an identifier of an annotator, an example and a class, saying an annotator $j$, an example $i$ and a class $k$. Because we focus on binary labeling problems, we map $c_{1}(k=1)$ and $c_{2}(k=2)$ to the negative ("-") and positive ("+") classes, respectively.

Each example $i$ associates a multiple noisy label set $\boldsymbol{l}_{i}=\left\{l_{i j}\right\}_{j=1}^{J}$, where every element $l_{i j}$ comes from annotator $j$. All labels of the examples in the dataset form an annotation matrix $L=\left\{\boldsymbol{I}_{i}\right\}_{i=i}^{I}, l_{i j} \in\left\{c_{1}, 0, c_{2}\right\}$, where 0 means that the annotator does not provide any label for that example. The model associates annotator $j$ with matrix $N^{(j)}=\left\{n_{i k}^{(j)}\right\}, \quad 1 \leq i \leq I$ and $1 \leq k \leq K$. Every element inside the matrix presents the number of times that annotator $j$ labels example $i$ to class $k$. In practice, each annotator labels an object at most once, that is, $n_{i k}^{(j)} \in\{0,1\}$. We also define two priori probabilities of the negative and positive classes as $p^{-}$and $p^{+}$. 
The goal is to estimate the gold standards $\hat{y}_{i}$ that minimize empirical error:

$$
R=\frac{1}{I} \sum_{i=1}^{I} \mathbf{I}\left(\hat{y}_{i} \neq y_{i}\right) \text {, given } L, y_{i} \text {, where } y_{i} \in\left(c_{1}, c_{2}\right) \text {. }
$$

$\mathbf{I}(\bullet)$ is an indicator function that outputs 1 when the test condition is satisfied; otherwise, it outputs 0 .

\subsection{Dawid and Skene's Algorithm (DS)}

DS [5], originally proposed by Dawid and Skene in the domain of medical diagnoses, introduces confusion matrices to model annotators. An element $\pi_{k l}^{(j)}$ within the confusion matrix of annotator $j$ is a probability of labeling examples with true class $k$ to class $l$.

E-step: Given an annotation matrix $L$, DS estimates the probabilities of each example $i$ belonging to a class $k$ by using the following equation:

$$
P\left(\hat{y}_{i}=c_{k} \mid L\right)=\frac{\prod_{J=1}^{J} \prod_{l=1}^{K}\left(\pi_{k l}^{(j)}\right)^{n_{i j}^{j}} P\left(c_{k}\right)}{\sum_{q=1}^{K} \prod_{j=1}^{J} \prod_{l=1}^{K}\left(\pi_{q l}^{(j)}\right)^{n_{i l}^{j}} P\left(c_{q}\right)}
$$

where $P\left(c_{k}\right)$ and $P\left(c_{q}\right)$ are estimated prior probabilities of classes $c_{k}$ and $c_{q}$, respectively, which are calculated in the M-step of the algorithm.

M-step: DS updates all confusion matrices of annotators and the priori probabilities of all classes.

$$
\begin{gathered}
\hat{\pi}_{k l}^{(j)}=\sum_{i=1}^{I} \mathbf{I}\left(\hat{y}_{i}=c_{k}\right) n_{i l}^{(j)} / \sum_{l=1}^{K} \sum_{i=1}^{I} \mathbf{I}\left(\hat{y}_{i}=c_{k}\right) n_{i l}^{(j)} \\
\hat{P}\left(c_{k}\right)=\frac{1}{I} \sum_{i=1}^{I} \mathbf{I}\left(\hat{y}_{i}=c_{k}\right)
\end{gathered}
$$

\subsection{GLAD}

GLAD [42] was proposed to model the expertise of each annotator $\left(\alpha_{j} \in(-\infty,+\infty)\right)$ and the difficulty level of each example $\left(1 / \beta_{i} \in[0,+\infty)\right)$. To estimate the labeling probability of an annotator $j$ on an example $i$, GLAD uses the following logistic model.

$$
P\left(l_{i j}=y_{i} \mid \alpha_{j}, \beta_{i}\right)=\frac{1}{1+e^{-\alpha_{j} \beta_{i}}}
$$

E-step: GLAD computes the posterior probabilities of both negative and positive classes of all examples given the values of two parameters $(\boldsymbol{\alpha}, \boldsymbol{\beta})$ from the last M-Step and the observed annotation matrix $L$.

$$
P\left(y_{i}=+\mid L, \boldsymbol{\alpha}, \boldsymbol{\beta}\right)=P\left(y_{i}="+" \mid \boldsymbol{l}_{i} \boldsymbol{\alpha}, \beta_{i}\right) \propto P\left(y_{i}="+"\right) \prod_{j=1}^{J} p\left(l_{i j} \mid y_{i}="+", \alpha_{j}, \beta_{i}\right)
$$

M-step: GLAD maximizes the standard auxiliary function $Q$ and updates the values of two parameters $(\boldsymbol{\alpha}, \boldsymbol{\beta})$ by using a gradient descent algorithm as follows:

$$
Q(\boldsymbol{\alpha}, \boldsymbol{\beta})=E[\ln P(L, \boldsymbol{y} \mid \boldsymbol{\alpha}, \boldsymbol{\beta})]=E\left[\ln \prod_{i=1}^{I}\left(P\left(y_{i}="+"\right) \prod_{j=1}^{J} P\left(l_{i j} \mid y_{i}="+", \alpha_{j}, \beta_{i}\right)\right)\right]
$$

where the vector $\boldsymbol{y}$ is the estimated class labels of all examples.

\subsection{Raykar, Yu and et al. (RY)}

RY [30] was proposed to model the sensitivity $\left(\alpha_{j}\right)$ and the specificity $\left(\beta_{j}\right)$ of an annotator $j$. In the case of binary labeling, the sensitivity defines the bias toward the positive class, and the specificity defines the bias toward the negative class. RY uses a classifier to predict labels, but this classifier 
requires a feature representation of examples. In our study, we ignore this classifier.

RY uses a Bayesian approach to estimate the priori probabilities of the parameters $\left(\alpha_{j}, \beta_{j}\right)$ and the positive class as follows.

$$
\begin{gathered}
P\left(\alpha_{j} \mid a_{j}^{+}, a_{j}^{-}\right)=\operatorname{Beta}\left(\alpha_{j} \mid a_{j}^{+}, a_{j}^{-}\right) \\
P\left(\beta_{j} \mid b_{j}^{+}, b_{j}^{-}\right)=\operatorname{Beta}\left(\beta_{j} \mid b_{j}^{+}, b_{j}^{-}\right) \\
P\left(p^{+} \mid n^{+}, n^{-}\right)=\operatorname{Beta}\left(p^{+} \mid n^{+}, n^{-}\right)
\end{gathered}
$$

where $a_{j}^{+}$and $a_{j}^{-}$are the number of positive labels and negative labels, respectively, provided by the annotator $j$ to the positive class inferred at this moment; $b_{j}^{+}$and $b_{j}^{-}$are the number of positive labels and negative labels, respectively, provided by the annotator $j$ to the negative class inferred at this moment; and $n^{+}$and $n^{-}$are the total number of positive and negative labels, respectively, provided by all annotators to all examples. Beta is a beta probability distribution function.

E-step: Given an example $\boldsymbol{x}_{i}$, an annotation matrix $L$, two parameters $(\boldsymbol{\alpha}, \boldsymbol{\beta})$ and the priori probability of the positive class $p^{+}$, RY computes the probability of each example $i$ belonging to the positive class as follows:

$$
\mu_{i}=P\left(y_{i}=+\mid \boldsymbol{x}_{i}, L, \boldsymbol{\alpha}, \boldsymbol{\beta}, p^{+}\right) \propto \frac{p^{+} a_{i}}{p^{+} a_{i}+\left(1-p^{+}\right) b_{i}}
$$

where

$$
\begin{aligned}
& a_{i}=\prod_{j=1}^{J}\left(\alpha_{j}\right)^{\mathbf{I}\left(L_{i j}="+"\right)}\left(1-\alpha_{j}\right)^{\mathbf{I}\left(L_{i j}="-"\right)} \\
& b_{i}=\prod_{j=1}^{J}\left(\beta_{j}\right)^{\mathbf{I}\left(L_{i j}="-"\right)}\left(1-\beta_{j}\right)^{\mathbf{I}\left(L_{i j}="+"\right)}
\end{aligned}
$$

$M$-step: RY updates the parameters $\left(\alpha_{j}, \beta_{j}\right)$ of annotator $j$ and the priori probability of the positive class $p^{+}$according to all observed labels provided by annotator $j$ (denoted by $\sum_{i} l_{i j}$ ) as follows:

$$
\begin{gathered}
\alpha_{j}=\frac{a_{j}^{+}-1+\sum_{i=1}^{I} \mu_{i} l_{i j}}{a_{j}^{+}+a_{j}^{-}-2-\sum_{i=1}^{I} \mu_{i}} \\
\beta_{j}=\frac{b_{j}^{+}-1+\sum_{i=1}^{I}\left(1-\mu_{i}\right)\left(1-l_{i j}\right)}{b_{j}^{+}-b_{j}^{-}-2+\sum_{i=1}^{I}\left(1-\mu_{i}\right)} \\
p^{+}=n^{+}-1+\sum_{i=1}^{I} \mu_{i} /\left(n^{+}-n^{-}-2+I\right)
\end{gathered}
$$

\subsection{ZenCrowd (ZC)}

ZenCrowd [6] only uses a binary parameter $\{\mathrm{good}, \mathrm{bad}\}$ to model the reliability of an annotator. It is slightly more complex than MV but simpler than the other above-mentioned methods. It is used to address the problem of entity linking for large collections of online pages.

E-step: Given all observed labels provided by each annotator $j$ (denoted by $\sum_{i} l_{i j}$ ), ZenCrowd calculates the reliability of annotator $j$, which is defined as follows:

$$
P\left(u_{j}=\text { reliable }\right)=\sum_{i=1}^{I} \mathbf{I}\left(l_{i j}=\hat{y}_{i}\right) / \sum_{k=1}^{K} \sum_{i=1}^{I} n_{i k}
$$


M-step: ZenCrowd uses reliabilities of annotators to update the probability of an example $i$ belonging to a specific class $c_{k}$.

$$
P\left(y_{i}=c_{k}\right)=\frac{\prod_{j=1}^{J}\left[P\left(u_{j}=\text { reliable }\right)\right]^{\mathbf{I}\left(\hat{y}_{i}=c_{k}\right)}}{\sum_{k=1}^{K} \prod_{k=1}^{w}\left[P\left(u_{j}=\text { reliable }\right)\right]^{\mathrm{I}\left(\hat{y}_{i}=c_{k}\right)}}
$$

\section{Adaptive Weighted Majority Voting Algorithm (AWMV)}

On the one hand, EM-based algorithms do not particularly consider bias; on the other hand, they have innate shortcomings because their initial parameter settings, which are not easy to set, strongly affect the results. In this section, a novel non-EM-based consensus algorithm for biased labeling is proposed.

Compared with EM-based algorithms (refer to experimental results in Section 5), MV does not necessarily perform worse. To the contrary, it is simple, robust and fast. As mentioned above, labeling bias is a systematic tendency, meaning that a large number of annotators have biases. A straightforward means of solving this bias issue is to adapt MV. If we assign different weights to different types of labels, the outcomes of integration will be shifted toward an expected direction. Specifically, if the weights assigned to the negative labels are less than are those assigned to the positive ones, more positive examples will be inferred. A key question is how to determine these weights.

\subsection{An Index to Describe the Bias}

In this subsection, we use a simulation to illustrate the unbiased and the biased labeling of annotators. For each annotator $j$, all labels obtained from $j$ are denoted by $\boldsymbol{l}^{(j)}$, the overall labeling quality, the labeling quality on the negative examples, and that on the positive ones are denoted by $p^{(j)}, p_{N}^{(j)}$, and $p_{P}^{(j)}$, respectively. These three types of labeling qualities can be estimated as follows.

$$
\begin{gathered}
p^{(j)}=P\left(l_{i j}=y_{i} \mid E, \boldsymbol{l}^{(j)}\right)=\frac{1}{I} \sum_{i=1}^{I} \mathbf{I}\left(l_{i j}=y_{i}\right) \\
p_{N}^{(j)}=P\left(l_{i j}=y_{i} \mid E, \boldsymbol{l}^{(j)}, y_{i}="-"\right)=\frac{1}{\sum_{i=1}^{I} \mathbf{I}\left(y_{i}="-"\right)} \sum_{i=1}^{I} \mathbf{I}\left(l_{i j}=y_{i} \& y_{i}="-"\right) \\
p_{P}^{(j)}=P\left(l_{i j}=y_{i} \mid E, \boldsymbol{l}^{(j)}, y_{i}="+"\right)=\frac{1}{\sum_{i=1}^{I} \mathbf{I}\left(y_{i}="+"\right)} \sum_{i=1}^{I} \mathbf{I}\left(l_{i j}=y_{i} \& y_{i}="+"\right)
\end{gathered}
$$

In our simulation, we assume that the labeling quality models of all annotators are consistent. Therefore, we omit the superscript $j$ in the following discussion. When labeling behaviors exhibit bias, the difference between $p_{N}$ and $p_{p}$ is significant. That is, $p_{N}-p_{p}>\delta$. In practice, we usually have $\delta \geq 0.2$.

We start with an unbiased situation in which annotators have the same error rates on both negative and positive examples. Most negative examples receive a large number of negative labels, and most positive ones receive a large number of positive labels. The frequency of positive labels of an example $e_{i}$ can be calculated as follows:

$$
f_{+}^{(i)}=\frac{n_{+}^{(i)}}{n_{+}^{(i)}+n_{-}^{(i)}}
$$

where $n_{+}^{(i)}$ and $n_{-}^{(i)}$ are the numbers of positive and negative labels, respectively, in the multiple 
noisy label set of example $e_{i}$. Supposing that the underlying class distribution is balanced, it is expected that most negative examples have their multiple label sets with $f_{+}<0.5$, and most positive examples have their multiple label sets with $f_{+} \geq 0.5$. We can cluster examples according to their $f_{+}$values to infer the potential truths of their class labels.

We simulate this unbiased situation as follows. Let all annotators have the same labeling quality $p$ $=0.7$. Because there are assumed no biases, both the labeling quality on the negative and that on the positive are the same (i.e., $p_{N}=p_{p}=p=0.7$ ). After all examples are labeled by nine annotators, we calculate $f_{+}$values of all examples and count the number of examples for every $f_{+}$value. Then, we normalize these counters to a range $[0,1]$ and plot the proportion of examples against the frequency of positive labels. Blue circle points in Fig. 1 represent the percentage of examples at each $f_{+}$value. If we fit these circle points to a curve (a blue dashed line), we can find two peaks and a valley on the curve. Peakl and Peak2 represent the maximum numbers of negative and positive examples at two different $f_{+}$values, respectively. The valley lies on the point with an x-coordinate of 0.5 , which is only the watershed of potential negative and positive examples. Because there is no bias, the curve is symmetric with respect to the line $x=0.5$. Fig. 1 is named a Positive Frequency Distribution (PFD) graph.

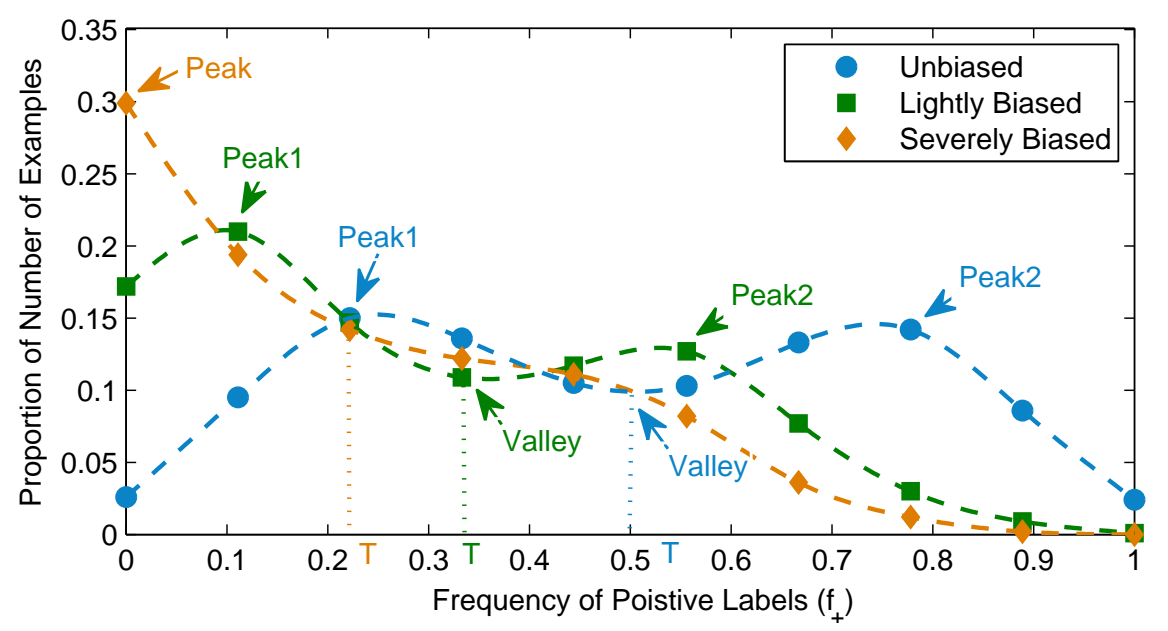

Fig. 1: Distributions of the proportion of examples against $f_{+}$under unbiased, lightly biased and severely biased conditions

When annotators have biases, they will provide more-negative labels on both negative and positive examples. Therefore, $f_{+}$of both negative and positive examples will decrease. Fig. 1 reflects this trend. Two peaks move toward the left. Green squares and their corresponding dashed curve represent the situation that the bias is not severe. At this point, the overall labeling quality remains 0.7 and $80 \%$ errors occur on the positive, i.e., $p_{N}=0.88$ and $p_{p}=0.52$. We still find two peaks and a valley on this curve. However, compared with the blue curve, the x-coordinate of the valley point decreases to 0.33. If the bias becomes more severe, positive labels occurring in the multiple label sets of positive examples become seriously scarce. The consequence is that the two peaks on the curve merge because the positive examples also have very small $f_{+}$values. We only find one unique maximum point on the curve, as orange diamonds and their corresponding dashed curve illustrate. At this point, the overall labeling quality remains 0.7 , but $90 \%$ errors occur on the positive examples, i.e., $p_{N}=0.94$ and $p_{p}=0.46$.

We utilize an index named Threshold of Positive Label Frequency $\left(f_{+T}\right)$ to describe the bias. If 
there is a valley on the curve, we treat the x-coordinate of the valley point as the index. Under the condition with no valley, we must find a point whose $\mathrm{x}$-coordinate is $T$ and draw a line $x=T$. If the area under the curve on the left of this line is almost the same as that on the right of this line, $T$ is the index. When $f_{+T} \approx 0.5$, there is no bias. The smaller $f_{+T}$, the more severe is the bias.

\subsection{Calculation of Bias Rate}

MV implicitly assigns the same weight 0.5 to both negative and positive labels, the summation of which equals 1. Under biased labeling situations, we set a bias rate $r$ to adjust the weights for the two classes as follows.

$$
\left\{\begin{array}{l}
w_{N}=(1-r)^{*} 0.5 \\
w_{P}=r^{*} 0.5
\end{array}, 0 \leq \mathrm{r} \leq 1\right.
$$

After we obtain two weights, the probability of an example $e_{i}$ to be negative and to be positive can be respectively estimated as follows.

$$
\left\{\begin{array}{l}
\operatorname{Pr}\left(\hat{y}_{i}={ }^{\prime}+'\right)=n_{+}^{(i)} w_{P} /\left(n_{+}^{(i)} w_{P}+n_{-}^{(i)} w_{N}\right) \\
\operatorname{Pr}\left(\hat{y}_{i}={ }^{\prime}-'\right)=n_{-}^{(i)} w_{N} /\left(n_{+}^{(i)} w_{P}+n_{-}^{(i)} w_{N}\right)
\end{array}\right.
$$

The final estimated class of this example is the one with the larger probability. How can we associate the bias rate $r$ with the threshold of the positive label frequency $\left(f_{+T}\right)$ ?

Intuitively, a smaller $f_{+T}$ results in a larger $r$. When $f_{+T}$ is set to 0.5 , the bias rate $r$ should be 0 . When $f_{+T}$ is set to 0 , the bias rate $r$ should be 1.0. Instead of employing a linear relationship between $r$ and $f_{+T}$, in which $r$ is inversely proportional to $f_{+T}$, we adopt non-linear functions to determine the relationship between $f_{+T}$ and $r$. According to our observation, when $f_{+T}$ is not too small (e.g., $f_{+T} \approx 0.4$ ), we hope that the speed of the increment of $r$ is greater than that of the decrement of $f_{+T}$; and when $f_{+T}$ is very small (e.g., $f_{+T} \approx 0.1$ ), we hope that the speed of the increment of $r$ is less than that of the decrement of $f_{+T}$. Thus, when the bias is not too severe, we assign a positive label a larger weight, so that the leverage inclines more toward positive labels. When the bias is severe, we assign a positive label a weight less than that calculated by the linear relationship to prevent those very large positive weights from overwhelming the small negative ones.

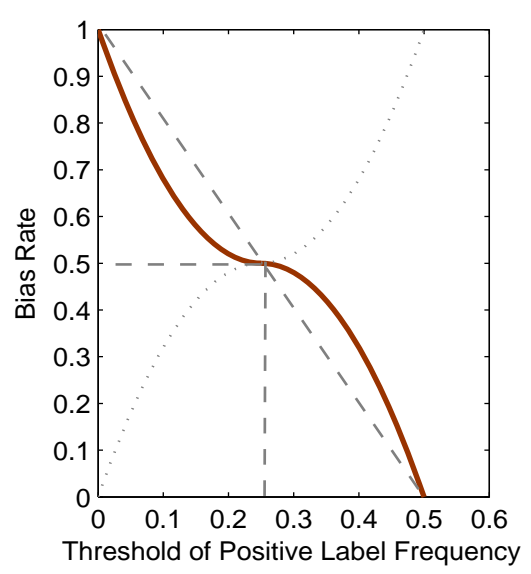

Fig. 2: Quadratic functions that map the threshold of the positive label frequency $f_{+T}$ to a bias rate $r$

We describe this relationship with the help of two quadratic functions. We let the vertices of these two quadratic functions overlap on the line $x=0.25$ so that their symmetrical domains can cover the 
interval [0, 0.5], shown in Fig. 2. The overlapped vertex of the two quadratic functions is the stationary point of the curve in dark red. The dark red curve is the bias rate $r$ with respect to $f_{+T}$. Given the stationary point $(0.25, \theta)$, we have four other points $(0,1),(0.5,1),(0,0)$ and $(0.5,0)$ to determine the analytic expressions of these two quadratic functions as follows.

$$
y= \begin{cases}16(1-\theta) x^{2}+8(\theta-1) x+1, & x \in[0,0.25] \\ -16 \theta x^{2}+8 \theta x, & x \in(0.25,0.5]\end{cases}
$$

In this study, we empirically set $\theta=0.5$. The bias rate $r$ with respect to $f_{+T}$ can be directly derived from (25) as follows.

$$
r= \begin{cases}8 f_{+T}^{2}-4 f_{+T}+1, & f_{+T} \in[0,0.25] \\ -8 f_{+T}^{2}+4 f_{+T}, & f_{+T} \in(0.25,0.5]\end{cases}
$$

Now, we focus on how to find the positive label frequency $f_{+T}$.

\subsection{Data Structure and Procedure for Finding $f_{+T}$}

To find the positive label frequency $f_{+T}$, we introduce a table, namely FreqTable. We initially calculate $f_{+}$for each example. Examples with the same $f_{+}$are grouped together. For each group, we create a row in FreqTable that contains three fields: a sequence number, an $f_{+}$value and the number of examples in this group. The sequence number ranges from 1 to the maximum number of groups. We can use either a sequence number or its $f_{+}$value to represent a corresponding row (group). We use a function count $\left(f_{+}\right)$to retrieve the number of examples belonging to the corresponding group.

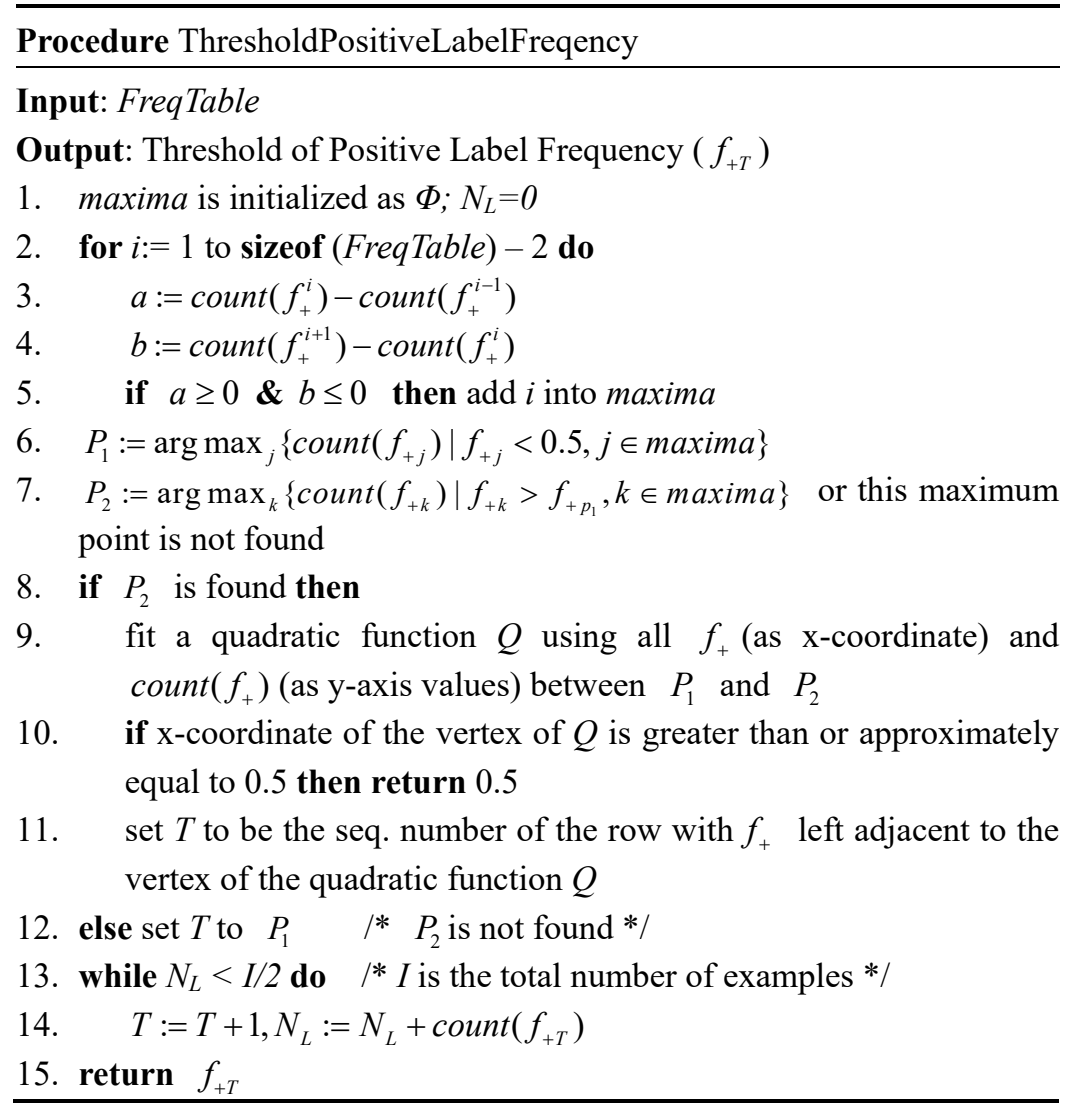

After FreqTable has been created, we sort the rows in the table in ascending order of $f_{+}$. 
Therefore, every row can be drawn as a point in Fig. 1. For each row, its $f_{+}$value is the x-coordinate, and the corresponding $\operatorname{count}\left(f_{+}\right)$is the y-coordinate. Then, procedure ThresholdPositiveLabelFreqency (shown above) is proposed to find the critical value $f_{+T}$ according to the sorted table.

The principle of Procedure ThresholdPositiveLabelFreqency is demonstrated in Fig. 1. The procedure uses an auxiliary set, namely maxima, to accommodate potential maximum points. After all potential maximum points (lines 1-5) are counted, the procedure tries to find two peaks (lines 6-7) from two auxiliary sets. If two peaks can be found, the green curve in Fig. 1 illustrates the situation. At this point, we fit all points between these two peaks using a quadratic function $Q$ (line 9). The vertex of $Q$ is a potential valley. However, if the x-coordinate of the vertex is greater than or approximately 0.5 , which means no bias is detected, we directly return 0.5 (line 10). Otherwise, we set the threshold $T$ to be the point (row) with $f_{+}$left adjacent to the vertex of $Q$ (line 12). If the second peak cannot be found, the orange curve in Fig. 1 illustrates the situation. At this point, we initially simply set the threshold $T$ to be the first peak (line 12). Then, we use other information to adjust $T$. The information utilized here is that when bias occurs, annotators tend to provide the label belonging to the major class. We use a variable $N_{L}$ to record the number of potential negative examples and let the number of potential negative examples be greater than that of positive ones (lines 13-14). After adjusting $T$, the procedure returns $f_{+T}$.

Now, we integrate all components of our proposed algorithm AWMV together, shown in its high-level pseudo code. At the beginning, the algorithm takes an entire dataset as its input. Note that every example in the dataset initially contains its multiple noisy label set but no integrated label. The output of the algorithm is that every example is assigned an integrated label. After AWMV has its input, the rows of FreqTable are sorted in ascending order of $f_{+}$(line 3). Then, the procedure above is called to find the positive label frequency $f_{+T}$ (line 4). After $f_{+T}$ has been determined, AWMV uses it to calculate a bias rate $r$ (line 5). The bias rate $r$ is used to calculate weights for two classes and then to infer an integrated label from the multiple noisy label set of each example (lines 6-9).

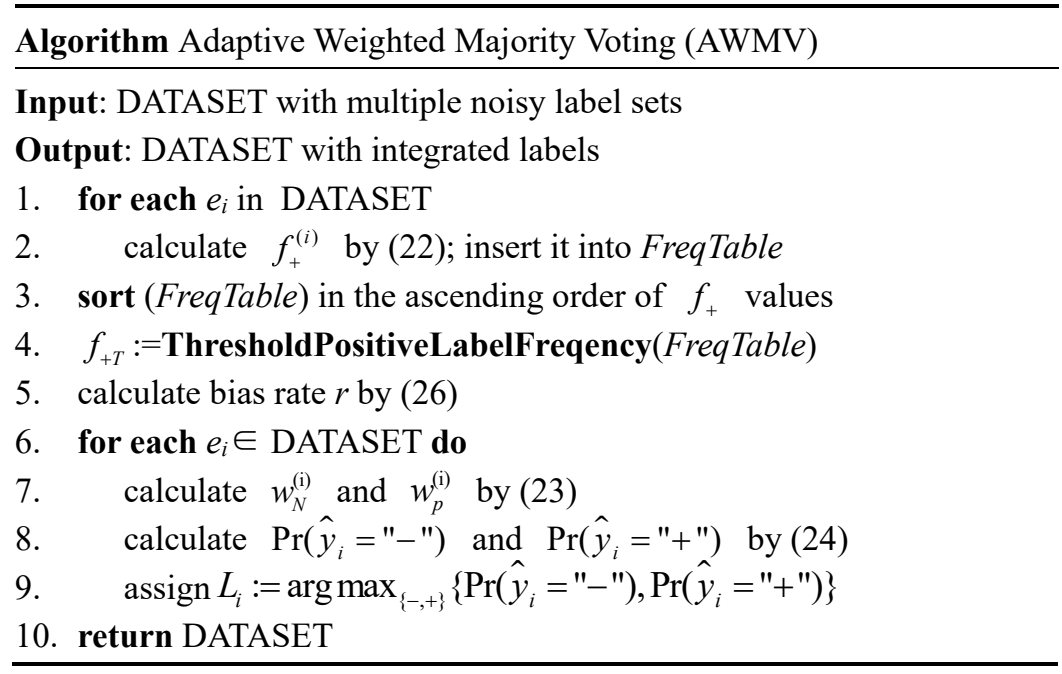

\subsection{Discussion of $A W M V$}

Generally, AWMV utilizes a heuristic strategy to search for the potential two peaks and the valley between them to determine whether biased labeling occurs and the extent of the bias. In most cases, there are two peaks on the $P F D$ graph (refer to Fig. 1). If the bias is very severe, the two peaks merge. 
We can use a simple binomial distribution to explain why and where we can find two peaks on the $P F D$ graph. In Section 4.1, we defined the biased labeling issue as $p_{N}-p_{p}>\delta$. Under an unbiased situation, e.g., both $p_{N}$ and $p_{P}$ are greater than 0.7 , because $1-p_{N}$ and $p_{p}$ are significantly different, two distinct peaks appear on the PFD graph. One, with an $f_{+}$value close to $0(<0.5)$, represents the center of negative examples. The other represents the center of positive examples. Given that the size of the multiple noisy label set of an example $e_{i}$ is $R$ ( $R=n_{+}+n_{-}$; we omit the superscript $i$ here for simplicity), the probability $\left(p_{k}\right)$ of having $k$ positive labels obeys a binomial distribution:

$$
\begin{gathered}
p_{k}^{+}=b\left(k ; R, p_{P}\right)=\left(\begin{array}{l}
R \\
k
\end{array}\right) p_{P}{ }^{k}\left(1-p_{P}\right)^{R-k}, \text { if } y_{i}="+" \\
p_{k}^{-}=b\left(k ; R, 1-p_{N}\right)=\left(\begin{array}{l}
R \\
k
\end{array}\right)\left(1-p_{N}\right)^{k} p_{N}{ }^{R-k}, \text { if } y_{i}="-"
\end{gathered}
$$

For a binomial distribution, the maximum of the probability density function occurs at $k$, which is the value of expectation. For the positive examples, the maximum of the probability density function occurs at $k=R \times p_{P}$ (i.e., $x=p_{P}$ on the $P F D$ graph); for the negative ones, the maximum of the probability density function occurs at $k=R \times\left(1-p_{N}\right)$. Thus, there must be two peaks on the $P F D$ graph. Between them, there is a valley. At this point, we have $p_{k}^{+}=p_{k}^{-}$, which means the following:

$$
b\left(k^{*} ; R, p_{P}\right)=b\left(k^{*} ; R, 1-p_{N}\right) \Rightarrow k^{*}=\frac{R\left(\log p_{N}-\log \left(1-p_{P}\right)\right)}{\log p_{P}+\log p_{N}-\log \left(1-p_{P}\right)-\log \left(1-p_{N}\right)}
$$

On the PFD graph, the valley occurs at $x=k^{*} / R$.

In the above discussion, we use a hypothetical setting as the simulation in Section 4.1. However, in a real-world condition, both $1-p_{N}$ and $p_{P}$ obey Gaussian distributions with different means and variances. A Gaussian distribution approximates a binomial distribution in which $R \rightarrow \infty$. Thus, using a binomial distribution is sound. Because both $1-p_{N}$ and $p_{P}$ obey two different Gaussian distributions, the distribution of all points on the $P F D$ graph conforms to the Gaussian Mixture Model (GMM). An EM approach is effective to estimate the parameters of GMM. However, the EM approach suffers from a defect. If two Gaussian distributions overlap too much, the accuracy of the estimation is very low. Under biased labeling situations, two Gaussian distributions often merge (i.e., two peaks on the PFD graph overlap). Therefore, the EM approach loses its effectiveness. AWMV takes another approach to solve this issue, determining the division of two Gaussian distributions by a heuristic search. Moreover, the EM approach tries to provide a cut point regardless of the level of overlapping of two Gaussian distributions.

Now, let us analyze the time complexity of these algorithms. For simplicity, we assume that the total number of examples is $I$, each example is labeled by $J$ annotators, and the number of classes is $k=2$. AWMV has two main steps. Its first step calculates $f_{+}$for all examples. Its second step usually involves one-half of the examples to calculate $f_{+T}$ and fits a quadratic function $Q$ (a quadratic fitting has a time complexity $O(4 N)$, where $N$ is number of points). Thus, the time complexity of AWMV is $O(5.5 \cdot I J)$. MV is the fastest, with time complexity $O(I J)$. For GLAD, ZC, and RY, in each iteration of EM, every label of each example is examined. Therefore, their time complexities are $O(T I J)$, where $T$ is the number of iterations of EM. In practice, $T$ is usually in the range $[10,50]$. Because DS additionally considers the confusion matrices of all annotators in each iteration, its time complexity is $O\left(T I J k^{2}\right)$, where $k=2$ for a binary labeling. Thus, AWMV has a smaller time complexity than all 
EM-based algorithms. Among these EM-based algorithms, DS is the most complex.

\section{Experiments and Analyses}

In this section, we will initially analyze immanent probabilistic patterns in real-world datasets in hopes of revealing traces of bias. Then, we will investigate the performance of the proposed algorithm AWMV compared with the four EM-based consensus algorithms described in Section 3 on eight real-world datasets and discuss their advantages and disadvantages under different scenarios.

\subsection{Real-World Datasets with Labeling Biases}

Recent work on crowdsourcing utilized MTurk as an online labor market system to elicit annotations from workers. All labels of the real-world datasets described in this section are collected via the MTurk platform. To support the targets of studies, the instances in these datasets are also labeled by human experts. These expert labels are treated as ground truths. Because the datasets were originally used for binary labeling, this paper only focuses on binary labeling. In general, bias means that annotators are prone to provide one type of label (possibly negative).

To depict the labeling behaviors of all non-expert annotators, we adopt the following metrics: average overall labeling quality $(\bar{p})$, average labeling quality on the negative $\left(\bar{p}_{N}\right)$ and on the positive $\left(\bar{p}_{P}\right)$, and the total number of negative labels (\#ln) and of positive labels (\#lp).

\subsubsection{Dataset Affective Text}

The original Affective Text dataset ${ }^{1}$ was constructed as an annotation task proposed by Strapparava et al. [37]. In their work, each annotator was asked to provide numeric judgments to rate the headline of a piece of news for several types of emotions and a numeric score, namely Valence, to rate the overall sentiment of this news. Snow et al. selected a 100-headline sample from the SemEval test set and collected 1000 affect labels for each label type ${ }^{2}$, in which each example was labeled by 10 unique annotators [35]. For each emotion, annotators were asked to provide scores in a range of [0, 100], and for Valence, the scores are in a range of $[-100,100]$. Following a coarse-grained 0/1 classification measure in [37], each emotion labeled by non-expert annotators with a score within $[0,50)$ or $[50,100]$ is respectively mapped to negative or positive. For binary labeling on Valance, we treat a score within $[-100,0]$ as negative or within $(0,100]$ as positive.

Table 1: Statistical Information of Eight Real-World Biased Datasets

\begin{tabular}{l|ccccc}
\hline dataset & $\overline{\boldsymbol{p}}(\boldsymbol{\%})$ & $\overline{\boldsymbol{p}}_{\boldsymbol{N}}(\boldsymbol{\%})$ & $\overline{\boldsymbol{p}}_{\boldsymbol{P}}(\boldsymbol{\%})$ & $\# \boldsymbol{n} / \# \boldsymbol{p}$ & $\# \ln / \# \boldsymbol{l} \boldsymbol{p}$ \\
\hline Fear & 80.8 & 94.5 & 22.6 & $79 / 21$ & $897 / 103$ \\
Valence & 65.0 & 87.3 & 41.5 & $50 / 50$ & $736 / 264$ \\
Adult & 74.6 & 71.5 & 46.9 & $187 / 146$ & $2106 / 1211$ \\
WordSim & 84.8 & 90.1 & 80.0 & $14 / 16$ & $158 / 142$ \\
Trec10 & 67.2 & 71.1 & 37.4 & $1774 / 1493$ & $12537 / 5938$ \\
Duck & 66.6 & 75.0 & 59.9 & $100 / 140$ & $5291 / 4309$ \\
BM & 49.1 & 58.8 & 26.2 & $490 / 510$ & $3594 / 1406$ \\
SpamCF & 69.4 & 91.9 & 5.10 & $69 / 31$ & $2119 / 178$ \\
\hline
\end{tabular}

Each example labeled by human experts also has a numeric score within a certain range. By manually analyzing these scores, we find the scale of the expert score is different from that of the non-experts. We extract two sub-datasets Fear and Valence from [35] and ask the experts in the domain

\footnotetext{
1 Available at: http://www.cse.unt.edu/ rada/affectivetext/\#datasets

2 Available at: https://sites.google.com/site/nlpannotations/
} 
of affective computing to re-label the data into two categories partially based on the median of the scores provided by the original datasets collected by Strapparava et al. [37]. The first two lines in Table 1 show the statistical quality information of the datasets Fear and Valence. We can observe that the labeling quality of the negative is significantly greater than that of the positive on these two datasets. The differences between these two labeling qualities are as high as 71.9 and 45.8.

Thus, the differences in scales or criteria between experts (gold values) and non-experts can lead to a systematic disparity of class labels. Under this condition, the integration of noisy labels hardly converges to a ground truth.

\subsubsection{Dataset Adult Content}

The dataset Adult Content ${ }^{3}$ (Adult) contains categories (G, PG, R, X) of the websites labeled by workers on MTurk reported in [16]. In that study, annotators had to examine web pages and classified them into four categories, depending upon the presence of adult content on the web pages. We remove inconsistent data as follows: (1) remove websites without ground truths; (2) remove websites that have never been labeled; and (3) remove annotators who labeled the websites without ground truths. The final dataset contains 269 annotators labeling 333 websites with 3317 annotations.

We classify all examples in the dataset into two categories: content without adult materials (labeled $\mathrm{G}$ ) as negative and content with adult materials (labeled PG, R, X) as positive. With the ground truths from human experts, the dataset contains 187 negative and 146 positive examples, whose underlying class distribution is balanced. We list the statistical information of the Adult dataset in Table 1. The labeling quality on the negative is clearly greater than that on the positive, and the difference between these two labeling qualities is as high as 24.6. The total number of negative labels from annotators is almost twice that of positive labels obtained (i.e., 2106:1211).

\subsubsection{Dataset Word Similarity}

The original dataset Word Similarity was used in [25], in which 30 word pairs were sorted in descending order of their contextual similarities. The word pairs were labeled by human experts in a real number range $[0,4]$. Snow et al. posted these word pairs on MTurk and collected 10 annotations for each word pair. The task was completed by 10 annotators with 300 labels [35].

In [25], word pairs sorted by similarities had the dividing cusp with an expert score of 2.17 , at which point the word pair "brother lad" with a score 2.17 was thought to be highly similar in most contexts. The next word pair with a score less than "brother lad" is "brother monk" (2.02), which is evidently not an approximate synonym. For binary classification, we classify word pairs with scores $[2.17,4]$ as true negative examples and with scores $[0,2.17)$ as true positive examples. The division is based on the following fact. For non-expert annotators, determining whether a pair of synonyms has a high contextual similarity is much easier than determining whether a pair of non-synonyms has a contextual similarity, because the synonym and the contextual similarity are not the same concepts. For annotations provided by non-experts, we deem the examples with scores $\in[0,5)$ positive and those with scores $\in[5,10]$ negative. Note that the score range in MTurk is in $[0,10]$.

The dataset $\mathrm{WordSim}^{4}$ has almost the same number of negative and positive examples. The statistical information of the dataset is listed in Table 1, showing that annotators still have a tendency to provide negative labels. The average labeling quality on negative examples is 10 greater than that on

\footnotetext{
3 Available at: https://github.com/ipeirotis/get-another-label/
}

4 Available at: https://sites.google.com/site/nlpannotations/ 
positive ones. However, at this point, both labeling qualities are greater than $50 \%$.

\subsubsection{Dataset Trec2010}

As a part of the TREC 2010 Relevance Feedback Track ${ }^{5}$ [40], non-expert annotators were provided a TREC format title, a description, and a narrative for each search topic using a pre-built judging interface [10]. They were asked to provide a judgment about whether the topics of web documents are relevant. We clean the data, removing the documents without ground truths (labeled "-1") and the documents whose links are broken (labeled "-2"). We treat the documents labeled "relevant" as negative and the documents labeled "non-relevant" as positive.

The dataset Trec $2010^{6}$ contains 3267 examples (1774 true negative ones and 1493 true positive ones) and 18,475 labels provided by 722 annotators (approximately 6 labels per example). We list the statistical information of the Trec10 dataset in Table 1. It shows that the annotators have a tendency to provide negative labels and that the labeling quality on the negative examples is approximately 33.7 greater than that on the positive ones. In this dataset, both the overall labeling quality and the labeling quality on the positive are quite low. It is the same as the dataset $A d u l t$, although the examples in this dataset contain different numbers of labels, ranging from one to over one thousand.

\subsubsection{Three Other Datasets Biased Binary Labeling}

Many binary labeling datasets are biased labeled. The dataset Duck contains binary judgments about whether a duck appears in a picture [41]. The dataset $B M^{7}$ includes labels $\{0,1\}$ from MTurk annotators for tweets, in which 0 is for negative and 1 for positive. It contains 83 annotators labeling 1000 examples. Its underlying class distribution is balanced, but the number of noisy labels on the negative is approximately twice that on the positive. The dataset $\operatorname{SpamCF}^{8}$ includes binary judgments about whether an HIT should be a "spam" [16]. It contains 150 annotators labeling 100 examples. The number of true negative examples is twice that of positive examples. However, the number of noisy negative labels is ten times greater than that of noisy positive labels. The labeling quality on positive examples is low to $5.1 \%$. The statistical information of these three datasets is also listed in Table 1 .

\subsubsection{Discussions on Biased Labeling}

The above analyses show that biased labeling is an innate attribute of crowdsourcing. Several causes can be discovered from these datasets. (1) Different measure scales between experts and non-experts. The bias in the datasets Fear and Valence resulted from this reason. (2) Lack of expertise. For example, lay people have insufficient professional knowledge on motion picture ratings. They cannot distinguish what type of content should be classified as Parental Guidance Suggested (PG) or Restricted (R). In the dataset Adult, many PG and R examples are easily classified as General (G) examples. (3) Deviant behaviors of annotators. The dataset SpamCF contains the task of identifying the spam behavior of an HIT task by reading the track log of this HIT task. The log contains so much information that most annotators do not have the patience to go over it but simply provide negative answers. Moreover, the preference of annotators is another type of deviant behavior.

The severity of biased labeling is related to underlying class distributions. However, the imbalance of underlying class distributions is not the premise of biased labeling. If an underlying class

\footnotetext{
5 Available at: https://www.ischool.utexas.edu/ ml/papers/trec-notebook-2010.pdf

6 Available at: http://trec.nist.gov/data/relevance.feedback10.html

7 Available at: http://people.csail.mit.edu/barzan/datasets/crowdsourcing/

8 Available at: https://github.com/ipeirotis/get-another-label/
} 
distribution is imbalanced, biased labeling occurs with a high probability (e.g., Fear, Adult and Spam $C F$ ). The imbalance of labeling is more serious than that of underlying class distributions. Table 1 shows that the ratio of negative and positive labels \#ln/\#lp is much greater than the number of negative and positive examples $\# n / \# p$ (e.g., Fear, Valence and SpamCF). Consequently, positive integrated labels become scarcer. We also notice that an underlying class distribution is occasionally almost balanced (e.g., WordSim, Valence, Trec10, and BM). Moreover, biased labeling also exists; for example, the ratio \#ln/\#lp for the balanced dataset $B M$ is 2.57 .

Biased labeling in crowdsourcing is a serious issue that can degrade the performance of existing unsupervised consensus algorithms. We will empirically study their performance on these biased datasets below.

\subsection{Experimental Setup}

Datasets and Tasks. The goal of our experiments is to evaluate the performance of five consensus algorithms and to investigate the effect of biased labeling on their performance. All experiments only are concerned with unsupervised estimation with no prior knowledge, although some algorithms claim that they gain better performance if more prior knowledge is provided [33]. We use accuracy, ROC Area and recall as evaluation metrics. All algorithms run on entire datasets to calculate the class labels of all examples. After calculation, the evaluation metrics are calculated against the ground truths. All eight datasets come from real-world applications and contain the biased labeling issue. We do not split a dataset into several parts because the goal of ground truth inference is to provide every example an integrated label, rather than developing training models and parameters for future predictions.

Implementations of Algorithms. For MV, RY and ZenCrowd, we use the codes implemented in SQUARE [33]. For GLAD, we use the code implemented by the authors of its original research article [42]. For DS, we use the code implemented by Ipeirotis [16]. During the experiments, we can add some auxiliary functions and change some control flow, but the core of each algorithm is not changed.

Initialization of parameters. The basic principle of addressing the initial values of algorithm parameters is to use their default values recommended by authors. Table 2 lists the default values of the parameters of the four EM-based algorithms and AWMV. In addition to these parameters, the priori probabilities of two classes are both set to 0.5 , and the probability of every example being positive is set to 0.5 . Initial settings can affect execution results. However, if we do not have prior knowledge, we must set the parameters based on the criterion that any class is treated impartially. The four EM-based algorithms stop when they converge. However, to avoid their target function vibrating around a local optimum, we also set a maximum number of iterations to each EM-based algorithm. In our experiments, the maximum iteration number is set to 50 , which is recommended by the authors of the corresponding implementations of these algorithms.

Table 2: Initial Settings of Algorithms

\begin{tabular}{l|l}
\hline Algorithm & Initial Values of Parameters \\
\hline MV & No Parameters \\
ZenCrowd & $P\left(u_{j}=\right.$ reliable $)=P\left(u_{j}=\right.$ unreliable $)=0.5$ \\
GLAD & $\alpha_{j}=\beta_{i}=1.0$ for all $1 \leq j \leq J$ and $1 \leq i \leq I$ \\
$\mathrm{RY}$ & $\alpha_{j}=\beta_{j}=0.5$ for all $1 \leq j \leq J$ \\
$\mathrm{DS}$ & $\pi_{p q}^{(j)}=0.9, p=q ; \pi_{p q}^{(j)}=0.1, p \neq q$ for all $1 \leq j \leq J$ \\
$\mathrm{AWMV}$ & No Parameters \\
\hline
\end{tabular}




\subsection{Comparison Results on Biased Labeled Datasets}

We conducted experiments on all biased datasets. Our comparison results are listed in Tables 3, 4 and 5 in terms of accuracy, ROC Area and milliseconds (running time), respectively.

\subsubsection{Comparison Results in Accuracy and ROC Area}

Tables 3 and 4 show our experimental results on eight biased datasets in terms of accuracy and ROC Area, respectively. The worst results are in italic. If there are multiple worst results, the figures are underlined. The best results are in bold. If there are multiple best results, the figures are also underlined.

Table 3: Accuracy on Eight Biased Datasets (\%)

\begin{tabular}{l|cccccc}
\hline Dataset & MV & ZC & GLAD & RY & DS & AWMV \\
\hline Fear & 81.0 & 80.0 & 82.0 & 82.0 & 83.0 & $\mathbf{8 6 . 0}$ \\
Valence & 68.0 & $\underline{67.0}$ & $\underline{67.0}$ & 75.0 & 74.0 & $\mathbf{8 0 . 0}$ \\
Adult & $\underline{84.4}$ & $\underline{84.4}$ & $\underline{84.4}$ & 88.0 & $\underline{84.4}$ & $\mathbf{8 8 . 7}$ \\
WordSim & 90.0 & $\underline{86.7}$ & $\underline{86.7}$ & $\underline{86.7}$ & 90.0 & $\mathbf{9 6 . 7}$ \\
Trec10 & 64.2 & 58.8 & 57.8 & 67.8 & $\mathbf{6 9 . 2}$ & 64.6 \\
Duck & 68.8 & 58.8 & 59.6 & 60.0 & 60.8 & $\mathbf{7 6 . 7}$ \\
BM & 49.7 & 49.8 & 49.3 & 50.3 & 50.7 & $\mathbf{5 1 . 4}$ \\
SpamCF & 66.0 & 66.0 & 66.0 & 66.0 & 66.0 & 66.0 \\
average & 71.5 & 68.9 & 69.1 & 72.0 & 72.3 & $\mathbf{7 6 . 3}$ \\
\hline
\end{tabular}

Table 4: ROC Area on Eight Biased Datasets

\begin{tabular}{l|cccccc}
\hline Dataset & MV & ZC & GLAD & RY & DS & AWMV \\
\hline Fear & 40.7 & 40.3 & 45.3 & 64.0 & 64.7 & $\mathbf{7 0 . 5}$ \\
Valence & 74.5 & 72.6 & 73.0 & 80.3 & 78.9 & $\mathbf{8 3 . 0}$ \\
Adult & 81.5 & 81.7 & 81.4 & 86.1 & 78.6 & $\mathbf{8 7 . 0}$ \\
WordSim & 85.3 & $\underline{83.0}$ & 83.3 & $\underline{83.0}$ & 85.3 & $\mathbf{9 2 . 9}$ \\
Trec10 & 66.3 & 61.1 & 60.3 & 70.0 & $\mathbf{7 1 . 2}$ & 67.4 \\
Duck & 70.4 & 61.6 & 62.4 & 62.5 & 63.5 & $\mathbf{7 7 . 3}$ \\
BM & 51.2 & 51.3 & 50.8 & 51.6 & 52.1 & $\mathbf{5 2 . 5}$ \\
SpamCF & 58.5 & 58.5 & 58.5 & 58.5 & 58.5 & 58.5 \\
\hline average & 66.1 & 63.8 & 64.3 & 69.5 & 69.1 & $\mathbf{7 3 . 6}$ \\
\hline
\end{tabular}

From Tables 3 and 4, we have the following observations. (1) The overall performance of the proposed AWMV is superior to that of all EM-based algorithms. Out of eight datasets, AWMV only performs worse than DS on Trec10. On average, the accuracy of AWMV is 4.1 greater than that of a runner-up DS. The ROC Area of AWMV is also 4.1 greater than that of a runner-up RY. (2) Among the four EM-based algorithms, both RY and DS have better performance. The performances of RY and DS have no significant difference on the real-world datasets with labeling bias, although RY introduces a Bayesian approach into its EM process. It appears that the Bayesian approach of the two parameters sensitivity and specificity cannot model the complexity of real scenarios very well. Both ZenCrowd and GLAD have poor performance, even worse than that of $\mathrm{MV}$, although their models are more complicated. (3) RY and DS are not better than MV on all datasets (e.g., not on WordSim and Duck), but AWMV is better than MV on all datasets. Thus, EM-based algorithms do not always have 
advantages over MV, but AWMV does because it is an enhanced version of MV.

To investigate the improvements in the qualities of the consensus algorithms, we compare the accuracy achieved in Table 3 with the values $\bar{p}$ in Table 1 . Note that Table 1 contains two types of datasets: datasets with relatively high labeling qualities (Fear, WordSim and Adult) and those with very low labeling qualities (Valence, Trec10, Duck, BM and SpamCF). For the datasets with high average labeling qualities (Fear, WordSim and Adult), the integrated labeling qualities of MV and of four EM-based algorithms are slightly greater than the average labeling qualities (Fear 80.8\%, WordSim $84.8 \%$ and Adult $74.6 \%$, as shown in Table 2). However, these increments are far away from their ideal ones. According to binominal theory [32], the labeling quality can be ideally improved up to $99.1 \%$, 99.4\% and 94.8\% for Fear, WordSim and Adult, respectively. For those datasets with low average labeling qualities (Trec10, Duck, BM and SpamCF), the four EM-based algorithms do not improve their labeling qualities at all. Both ZenCrowd and GLAD actually perform worse than their average labeling qualities (Trec10 67.2\%, Duck 66.6\%, BM 49.1\% and SpamCF 69.4\%, as shown in Table 1).

From the above analysis, we can draw the following conclusions. (1) The effectiveness of the four existing EM-based algorithms in improving the integrated labeling quality is very limited when the average labeling quality is moderate $(70 \% \sim 85 \%)$. When the average labeling quality is relatively low, they could not improve the integrated labeling quality at all, and occasionally even perform worse than average labeling qualities. (2) Compared with the EM-based algorithms, AWMV can improve the integrated labeling quality much more.

\subsubsection{Comparison Results in Running Time}

We investigate the time consumption of AWMV compared with the others. All algorithms are implemented in Java. We tested them on a PC with Intel(R) Core i5(TM)-5200U $2.20 \mathrm{~Hz}$ 2-core CPU, 8GB RAM, Java 7 VM and 64-bit Windows 7. The running time of each algorithm excludes any data load overhead. Table 5 lists the running time in milliseconds of each algorithm on all datasets.

Table 5: Running Time on Eight Biased Datasets (in milliseconds)

\begin{tabular}{l|rrcrrr}
\hline Dataset & MV & ZC & GLAD & RY & DS & AWMV \\
\hline Fear & 5 & 292 & 336 & 267 & 1495 & $\mathbf{9 0}$ \\
Valence & 6 & 275 & 343 & 237 & 1336 & $\mathbf{8 9}$ \\
Adult & 8 & 790 & 748 & 626 & 2095 & $\mathbf{1 3 4}$ \\
WordSim & 2 & 158 & 318 & 154 & 615 & $\mathbf{8 8}$ \\
Trec10 & 23 & 4992 & 3457 & 4856 & 9102 & $\mathbf{1 5 9}$ \\
Duck & 9 & 725 & 832 & 576 & 4379 & $\mathbf{8 5}$ \\
BM & 12 & 1103 & 851 & 768 & 2142 & $\mathbf{1 1 2}$ \\
SpamCF & 4 & 484 & 311 & 365 & 884 & $\mathbf{6 5}$ \\
average & 8 & 1102 & 899 & 981 & 2756 & $\mathbf{1 0 3}$ \\
\hline
\end{tabular}

MV undoubtedly has the lowest time cost. Its running time is approximately several to dozens of milliseconds on all datasets. The running time of AWMV is approximately dozens to several hundreds of milliseconds. The running times of ZC, GLAD, and RY are at the same level, which are approximately hundreds to several thousands of milliseconds. GLAD and RY are better than ZC because they transform continuous multiplications to continuous additions by logarithmic calculation. DS has the longest running time; it is the most complicated model, with enormous continuous 
multiplications. Obviously, the running time of AWMV is empirically more than ten times faster than the running times of the EM-based algorithms. These observations show high consistency with the theoretical complexity analysis of these algorithms in Section 4.4.

\subsection{Detailed Analysis of Results on Typical Datasets}

Tables 3 and 4 show that the performance of ZenCrowd and GLAD cannot even win against the baseline MV. Some datasets (i.e., Fear, Valence, Adult, BM, and SpamCF) have almost the same performance as MV does, with the maximum difference at only one point. On other datasets, WordSim, Trec10, and Duck, the performance of these two EM-based algorithms is even worse. Why do these algorithms with significantly different internal mechanisms obtain similar outcomes? Let us analyze the potential reasons using two typical datasets Valence and Trec10.
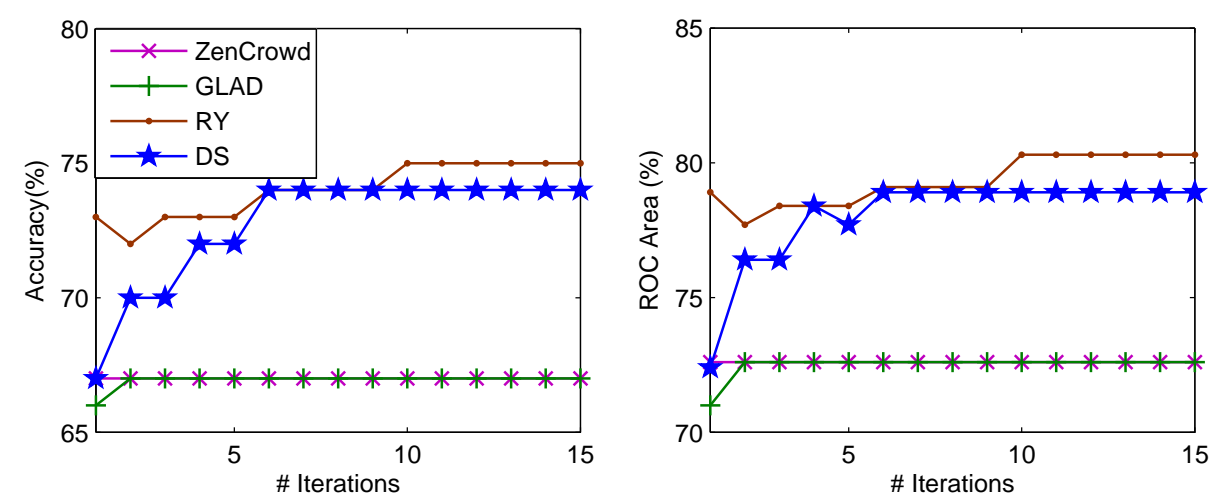

Fig. 3: Accuracy and the ROC Area under each iteration of the four EM-based algorithms. On the valence dataset, we set the compulsive number of iterations to be 15 , under which RY gains the best performance.

\subsubsection{Performance Analysis on Valence}

We conducted a further investigation on the Valence dataset, which has a balanced underlying data distribution. We traced whether the performance of ground truth estimation is improved under each iteration with respect to the four EM-based algorithms. At the end of each iteration, we evaluated their performance (in both accuracy and ROC Area) against the ground truths and recorded the values of modeled parameters. To avoid algorithms exiting early, we keep running algorithms until a given number of iterations was reached. We set the compulsory number of iterations to be 15 .

Fig. 3 shows the accuracy and the ROC Area of the four algorithms at the end of each iteration. The performance of both ZenCrowd and GLAD kept no changes after the second iteration. Both RY and DS showed expected behaviors. They converged once they reached their best performance after several iterations.

Fig. 3 shows that the EM procedure of ZenCrowd does not make any progress on improving the ground truth estimation. To validate this point of view, we trace the reliabilities (the model parameters) of all annotators in each iteration. We only plot the values of the first four iterations of the first 38 annotators, shown in Fig. 4. To show results more intuitively, we sort all annotators according to the ascending order of reliabilities in the first iteration. Thus, if there is a change of reliabilities of the annotators in each round of an iteration, we can observe a fluctuant plane. Fig. 4 shows that these values vary slightly between the second iteration and the first iteration. After the second iteration, the reliabilities of all annotators never change again. This observation is consistent with the results shown in Fig. 3. 


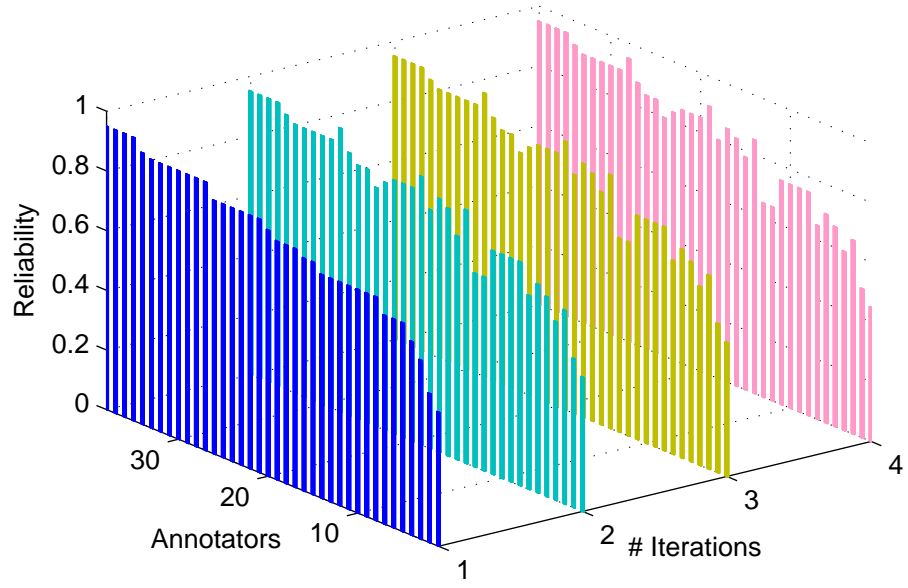

Fig. 4: The reliabilities of 38 annotators in the first four iterations of ZenCrowd. Every bar in the same plane presents the reliability value of an annotator. From the second iteration, the reliability value of every annotator is never changed. The effectiveness of ZenCrowd is equivalent to that of MV.

Why does ZenCrowd produce the same accuracy result in each iteration, almost as MV does? At the beginning, ZenCrowd uses a majority voting procedure to infer the estimated label for each example with an initial probability. The probability is the frequency of the estimated label appearing in the multiple label set of the example. After the initial procedure, only 18 examples (100 in total) are estimated as positive ones.

In E-Step, ZenCrowd calculates the reliability of each annotator using (17). In M-Step, ZenCrowd uses reliabilities of annotators to update the probability that an example belongs to a specific class using (18). In the initial step and the first round of E-Step, ZenCrowd uses majority voting to calculate the reliabilities of annotators. Therefore, if the number of negative labels in the multiple label set of an example outnumbers that of its positive labels, this example should be estimated as negative. Then, in M-Step, we still have the following:

$$
\prod_{j=1}^{J}\left[P\left(u_{j}=\text { reliable }\right)\right]^{\mathrm{I}\left(\hat{y}_{i}="-"\right)} \geq \prod_{j=1}^{J}\left[P\left(u_{j}=\text { reliable }\right)\right]^{\mathrm{I}\left(\hat{y}_{i}="+"\right)}
$$

According to (18), after the probabilities are updated, this example should remain estimated as negative. This situation also occurs for an example whose estimated label is positive in the first round of E-Step. Thus, in the next iteration of E-Step, ZenCrowd uses the same estimated labels of all examples to update all reliabilities of annotators. Therefore, nothing is changed. Overall, the EM procedure of ZenCrowd does not minimize the objective on this dataset.

GLAD behaves similarly to ZenCrowd. We traced the values of its two parameters $\left(\alpha_{j}, \beta_{i}\right)$ and its standard auxiliary function $Q$ at the end of each iteration. To present the traced data more clearly and straightforwardly, we define the following variables.

Definition (accumulative difference of $\alpha_{j}$ between two iterations $p$ and $q$ ) is the summation of the differences of parameter $\alpha$ s of all annotators.

$$
\Delta \boldsymbol{\alpha}^{(p, q)}=\sum_{j=1}^{J}\left|\alpha_{j}^{q}-\alpha_{j}^{p}\right|
$$

Definition (accumulative difference of $\beta_{i}$ between two iterations $p$ and $q$ ) is the summation of the differences of parameter $\beta$ s of all examples. 


$$
\Delta \boldsymbol{\beta}^{(p, q)}=\sum_{i=1}^{I}\left|\beta_{i}^{q}-\beta_{i}^{p}\right|
$$

Table 6 lists the above variable values under the first 10 iterations of GLAD. This table shows that after the second iteration, GLAD has already converged. Although the values of its parameters still vary, the magnitude of changes becomes smaller and smaller. These tiny changes are not sufficient to change the final results of the ground truth estimation of examples. The EM procedure is hovering at a local optimal point. To validate our supposition, we increase the number of iterations to 300 . The value of $Q$ eventually stabilizes at "-586.815" after the 202nd iteration, which is only 0.215 greater than its value at the 10th iteration. This result occurs because this biased dataset drives the GLAD algorithm to converge rapidly to a local optimal point.

Table 6: Variety of Parameters under the First 10 Iterations

\begin{tabular}{c|lll}
\hline Iteration & $\Delta \boldsymbol{\alpha}^{(\boldsymbol{p}, \boldsymbol{p})}$ & $\Delta \boldsymbol{\beta}^{(\boldsymbol{p}+\boldsymbol{p} \boldsymbol{p})}$ & $\boldsymbol{Q}^{(\boldsymbol{p + 1 , p )}}$ \\
\hline 1 & 12.90 & 16.47 & -621.947 \\
2 & 6.282 & 41.08 & -584.999 \\
3 & 0.336 & 0.325 & -587.166 \\
4 & 0.122 & 0.176 & -587.171 \\
5 & 0.116 & 0.210 & -587.129 \\
6 & 0.083 & 0.169 & -587.117 \\
7 & 0.088 & 0.188 & -587.081 \\
8 & 0.071 & 0.161 & -587.070 \\
9 & 0.074 & 0.169 & -587.038 \\
10 & 0.063 & 0.153 & -587.030 \\
\hline
\end{tabular}

In the original implementation of GLAD, its stop criterion is $\mid(Q-$ last $Q) /$ last $Q \mid>1.0 E-5$. After four iterations, this criterion is satisfied; GLAD then stops. If we adopt the convergence as a stop criterion in the four EM-based algorithms, rather than enforcing them to run a certain number of iterations, RY converges after the 10th iteration, and DS converges after the 8th iteration. However, both GLAD and ZenCrowd converge after the second iteration. That is, the EM mechanism in GLAD and ZenCrowd does not take effect.

\subsubsection{Performance Analysis on Trec10}

Among the eight datasets in Table 1, Trec10 is another typical dataset that has a moderate average labeling quality, a varied number of labels on examples and a conspicuous level of bias. Its notable feature is that some examples have many labels, whereas others have only one or two labels.

We traced the performance of the four algorithms after every iteration of EM. Fig. 5 shows the results of the four algorithms in terms of both accuracy and ROC Area. We find that at the beginning, ZenCrowd, GLAD and DS perform the same as MV does, whereas the performance of RY is obviously greater than that of MV. Then, both ZenCrowd and GLAD degrade steadily as the number of iterations increases. At the end, both of them converge at the minimum accuracy and ROC Area value, which is much lower than their performance obtained at the beginning. The performance of RY increases after the second iteration, but then there is no more improvement. It converges very quickly. Only DS maintains its improvement stability; its performance keeps increasing until the 12 th iteration, reaching 69.2\%. However, its increment is far from an ideal one. According to binominal theory [32], the labeling quality can be ideally improved up to $83.5 \%$ for Trec10 (its average labeling quality is $67.2 \%$ in Table 1). 

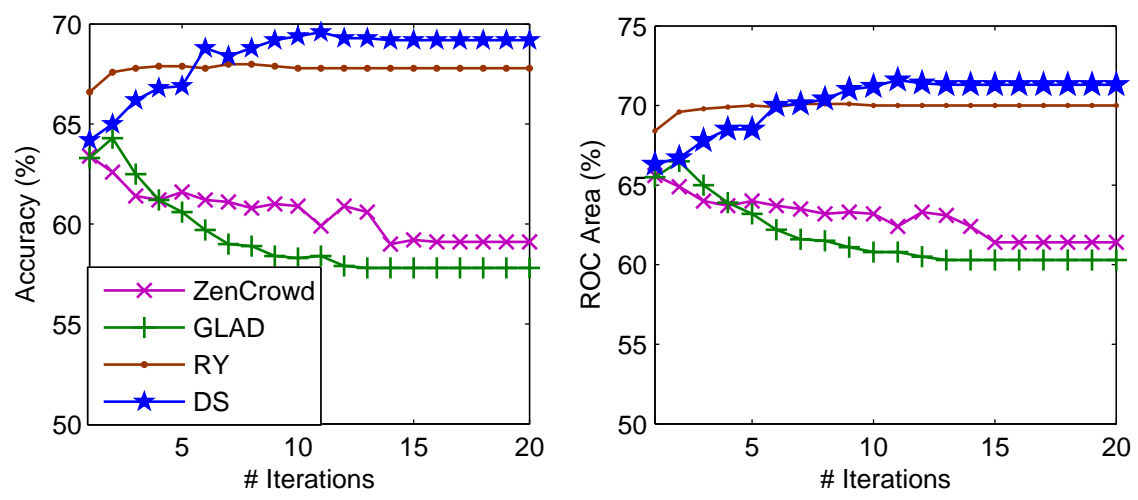

Fig. 5: Accuracy and the ROC Area of four algorithms after each iteration of the EM procedure. On the Trec10 dataset, we set the compulsive number of iterations to 20. DS is superior to others.

In many cases, for example on the Fear, Valence and Adult datasets, RY is slightly superior to DS, with an improvement of approximately $1 \%$ to $2 \%$. However, on the datasets with a lower quality and a high level of complexity (such as the Duck, Trec10, BM and SpamCF datasets), compared with DS, RY does not show any advantage, and is even inferior to DS. One reason might be that RY applies a Beta distribution to estimate the probabilities of parameters $\alpha_{j}$ and $\beta_{j}$. Beta distribution contains two parameters $a$ and $b$. From the graph of the Beta distribution, we can see that when $a$ and $b$ are small integers and the difference between these two parameters is large, its probability density is high and the probabilities of $\alpha_{j}$ and $\beta_{j}$ are large. However, there are many examples with almost the same number of negative and positive labels (corresponding to $a$ and $b$ ) in the Trec10 dataset; RY loses its advantage of using Bayesian estimation on this type of dataset.

Therefore, the complexity of a dataset really is a serious issue for EM-based algorithms. Although DS and RY outperform GLAD and ZenCrowd, their increment is only approximately $2 \%$, far from $20 \%$ (the minimum improvement according to binominal theory [32]).

\subsection{Effect of Bias}

In this section, we investigate the minority discovering abilities of the six algorithms. We use recall to evaluate the ability of an algorithm to infer positive examples correctly. Recall is also defined as a true positive rate, which is the proportion of true positive examples that an algorithm infers correctly. Table 7 shows the recall values of all algorithms on the eight real-world biased datasets. We observe the following from this table. (1) Among all six algorithms, our AWMV achieves the highest average recall rate, which is 11.5 greater than that of runner-up RY. (2) Among these four EM-based algorithms, RY achieves the highest average recall rate. DS is slightly inferior to RY. MV, ZenCrowd and GLAD have poor average recall rates. On three datasets with a moderate level of bias (Valence, Adult and WordSim), RY is slightly greater than DS on the dataset Adult, but is slightly lower than DS on the dataset WordSim. On the five complex and seriously biased datasets (i.e., Fear, Duck, Trec10, BM and Spam CF), RY and DS have almost the same recall rates. RY's ability to infer correctly the positive does not manifest. On these four datasets, all recall rates of the EM-based algorithms are very low. In particular, on the dataset SpamCF, only three positive examples are correctly inferred by all algorithms. Note that it actually has 31 true positive examples. (3) Although both AWMV and RY have relatively high recall values, on average, their recall rates remain at a low level $(61.0 \%$ for AWMV and $49.5 \%$ for $\mathrm{RY})$. 
Table 7: Recall on the Eight Biased Datasets (\%)

\begin{tabular}{l|cccccc}
\hline Dataset & MV & ZC & GLAD & RY & DS & AWMV \\
\hline Fear & 14.3 & 9.5 & 14.3 & 47.6 & 47.6 & $\mathbf{5 2 . 4}$ \\
Valence & $\underline{36.0}$ & $\underline{36.0}$ & $\underline{36.0}$ & 54.0 & 52.0 & $\mathbf{6 6 . 0}$ \\
Adult & 69.2 & 70.5 & 68.5 & 78.8 & 69.2 & $\mathbf{8 9 . 7}$ \\
WordSim & 87.5 & 81.3 & 81.3 & 81.2 & 87.5 & $\mathbf{1 0 0}$ \\
Trec10 & 38.7 & 15.8 & 11.0 & 62.2 & 59.2 & $\mathbf{6 5 . 1}$ \\
Duck & 51.5 & 30.0 & 31.4 & 32.1 & 34.3 & $\mathbf{6 8 . 6}$ \\
BM & 26.2 & 26.2 & 25.7 & 30.2 & 30.8 & $\mathbf{3 7 . 1}$ \\
SpamCF & 9.68 & 9.68 & 9.68 & 9.68 & 9.68 & 9.68 \\
average & 41.6 & 34.9 & 34.7 & 49.5 & 48.8 & $\mathbf{6 1 . 0}$ \\
\hline
\end{tabular}

According to our understanding, there are only two main factors that affect the recall rate- the level of bias and the overall labeling quality. Other external factors such as the difficulties of objects are ultimately reflected by these two factors. Given ground truths, we can define an index named Level of Imbalance (LI) as follows, which describes the difficulty of the biased dataset to an algorithm.

$$
L I=\frac{\bar{p}_{N}}{\bar{p}_{P}} \cdot \frac{1}{\bar{p}}=\frac{\sum_{j=1}^{J} \pi_{11}^{(j)}}{\sum_{j=1}^{J} \pi_{22}^{(j)}} \cdot \frac{I}{\sum_{j=1}^{J}\left(\pi_{11}^{(j)} \sum_{i=1}^{I} n_{i 1}^{(j)}+\pi_{11}^{(j)} \sum_{i=1}^{I} n_{i 2}^{(j)}\right)}
$$

We calculate the $L I$ values of all biased datasets mentioned above and sort them in ascending order. After we sort these datasets, we find that the recall rates of algorithms follow a rule; the recall rates decline as the $L I$ values increase. Fig. 6 shows this tendency clearly, particularly for AWMV, RY, DS and MV. With the increment of $L I$ values of the datasets (a dark gray dashed line in the figure), the recall rate becomes smaller and smaller (except the Duck dataset and the Fear dataset on some algorithms). Although the existing EM-based algorithms establish the varieties of models, they still do not solve the biased issue. However, our AWMV makes a certain progress.

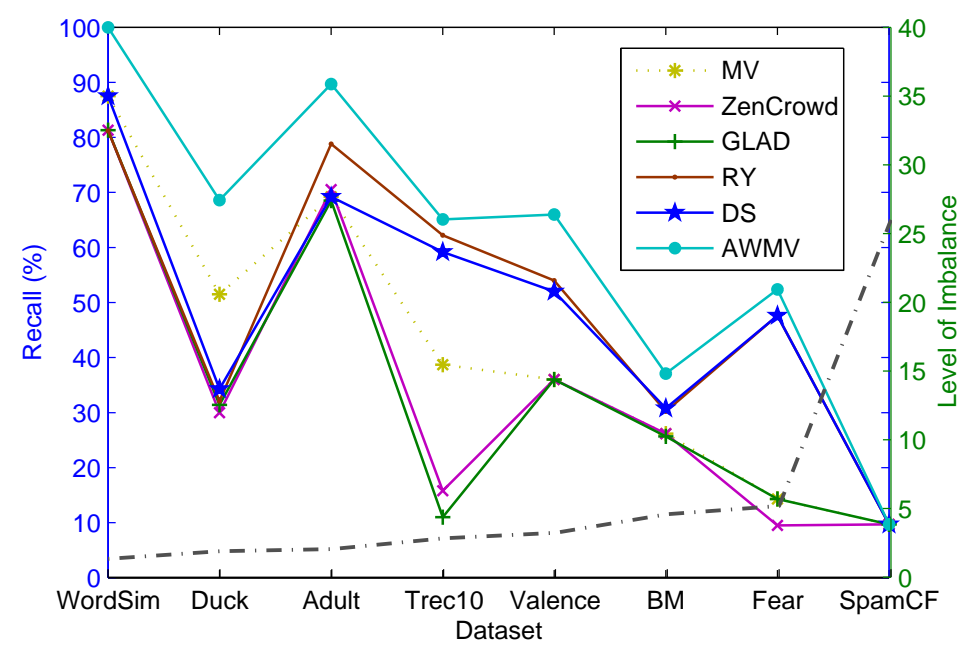

Fig. 6: The recall rates of six algorithms against the Level of Imbalance (LI). Datasets are sorted in ascending order of $L I$ values.

\subsection{Effect on Models Learned}

Because we mentioned in Section 1 that biased labeling affects the subsequent model learning process if inferred data are used for model training, in this section we investigate the effect of biased labeling on the performance of learned models. Because the above eight datasets are all only suitable for studying inference algorithms (without proper features), we had to collect a proper dataset from 
MTurk. We randomly chose 500 negative and 500 positive examples from a UCI dataset about "predicting whether a person's income exceeds \$50K/year based on the 1994 census data." 9 We posted this 1000-instance dataset on MTurk and required 15 workers to make predictions. Every instance was exactly labeled by the 15 workers.

After collecting the labels, the dataset has the following characteristics: $\bar{p}=64.1 \%$, $\bar{p}_{N}=79.3 \%, \bar{p}_{P}=48.8 \%$ and $\# n / \# p=500 / 500$. Obviously, the workers tend to make conservative predictions (an annual income is less than or equal to $\$ 50 \mathrm{~K}$ ). We named this dataset income1000. Here, we conducted a set of more elaborate experiments as follows. We conducted 15 groups of experiments. For these groups, the number of labels on each instance increases from 1 to 15 . For each instance, the labels used in experiments are randomly chosen from its 15-label set. After labels on each instance are chosen, a ground-truth inference algorithm is applied on this dataset to assign every instance an inferred class label. Then, we used these inferred labels of instances and their features to build the models. Although there are several classification algorithms that can be chosen for model training such as logistic regression, SVM [11] [12] [13], and neutral networks, we choose the decision tree which is more suitable for this dataset. A 10-fold cross validation is used to evaluate the performance of the learned models. Every group of experiments repeats 10 times, and the average performance in terms of ROC Area is reported.

We plotted the model performance after applying each of the six inference algorithms against different numbers of labels per instance as a learning curve shown in Fig. 7. When the number of labels on each instance is greater than three, the biased labeling issue causes MV, ZenCrowd, and GLAD to perform poorly. DS takes effect after the number of labels per instance reaches eight. AWMV and RY take effect after the number of labels per instance reaches three. When the number of labels per instance is greater than eight, the tendency of the performance of AWMV, RY and DS are similar, but the performance of AWMV is obviously better than that of RY and DS.

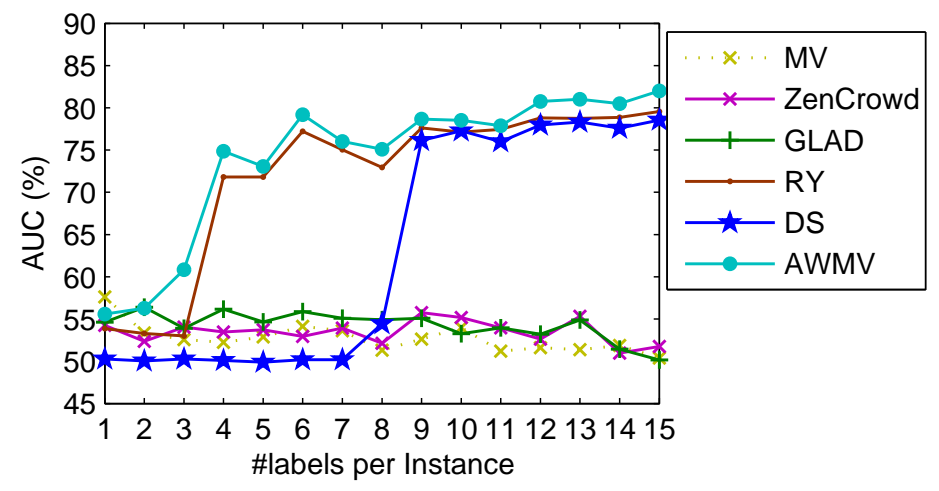

Fig. 7: Learning curves (after ground truth inference by six different algorithms) of 10 -fold cross validation using J48 as a base classifier on the income1000 dataset

\section{Conclusions}

When non-expert annotators label objects via crowdsourcing systems, biases could not be avoided due to the lack of expertise, different measure scales or individual preferences. This paper aims to arouse research communities' attention on the biased labeling issue in crowdsourcing and our solution to this issue. We analyzed several real-world crowdsourcing datasets collected from different application domains and revealed that the biased wisdom of the crowd is a tangible, widespread

9 Available at: http://archive.ics.uci.edu/ml/datasets/Adult 
problem. When biased labeling exists, although the overall labeling qualities of annotators can be relatively high, the labeling quality on a certain class (assuming the positive one) can remain quite low.

To investigate this biased labeling issue thoroughly, we empirically study four existing EM-based consensus algorithms: DS, GLAD, RY and ZenCrowd, which model different aspects of crowdsourcing systems, and our proposed non-EM-based algorithm adaptive weighted majority voting (AWMV). We compared them in terms of accuracy, ROC Area and recall on different biased real-world datasets and analyzed their performance in detail. Our experimental results show that biased labeling makes it difficult for all state-of-the-art EM-based consensus algorithms to infer the true class of potential positive examples. Among all compared algorithms, our proposed AWMV has the best overall performance. For those EM-based algorithms, both GLAD and ZenCrowd obviously cannot address biased labeled datasets and have very poor performance that is inferior even to the baseline MV; the classical DS and its Bayesian version RY have a better performance than do GLAD and ZenCrowd. However, both DS and RY can only improve the average labeling quality slightly, far from the ideal increment given by binominal theory [32].

EM-based algorithms are thought to be the most efficient algorithms on unsupervised consensus, but our experimental results show that on most biased datasets, even the best algorithm RY cannot achieve an average recall rate of $50 \%$. Although the recall rate of our proposed AWMV achieves approximately 10 points greater than RY does, these inference algorithms still face challenges when addressing biased and complex datasets in which more-important positive data points cannot be efficiently inferred.

There are many interesting research topics untouched which are related to the biased wisdom of the crowd and can arouse more research enthusiasm in research communities. Here is a list of some potential research issues.

- General ground truth inference algorithms might not be suitable for some special domains such as SpamCF. In these types of domains, a small portion of annotators might be experts. It must detect and assign these annotators a higher priority.

- Many real-world applications are cost-sensitive. Under cost-sensitive environments, misclassification costs are different. Cost-sensitive ground truth inference and learning is another interesting issue that must be studied.

- Injecting the ground truths of a small amount of examples to improve EM-based algorithms is an interesting research topic and must be studied.

- Current research on crowdsourcing focuses on binary labeling. Many real-world applications are multi-class. It is necessary to study ground-truth inference algorithms for multi-class noisy labeling. GLAD only works for binary domains. Although the authors of RY discussed its extension for multi-class scenarios, no detailed algorithm or implementation is published. Although ZenCrowd and DS can be directly applied to multi-class, deeper studies remain unlaunched.

- Multi-class noisy labeling is a great challenge. Reducing multi-class noisy labels to multiple noisy binary labels is attractive and faces many different choices. When labels are biased and noisy, problems become more complex.

- All current studies implicitly have an assumption that if a consensus algorithm makes more-correct inferences, subsequent machine learning algorithms achieve higher performance. However, this assumption does not always hold. For example, only when 
supported vectors are correctly inferred can SVM achieve high classification performance.

All datasets are available at http://sun0.cs.uca.edu/ ssheng/DA/datasets/BiasedDatasets.zip.

\section{Acknowledgments}

The authors thank anonymous reviewers for their insightful and constructive comments that have helped improve the quality of this paper and also thank Professor John Boyland from University of Wisconsin at Milwaukee for helping us improve the English presentation. This research has been supported by the National Natural Science Foundation of China under Grant No. 61603186, the Natural Science Foundation of Jiangsu Province, China, under Grant No. BK20160843, the China Postdoctoral Science Foundation under Grant No. 2016M590457, the Postdoctoral Science Foundation of Jiangsu Province, China under Grant No. 1601199C, the Project supported by the Jiangsu Key Laboratory of Image and Video Understanding for Social Safety (Nanjing University of Science and Technology) under Grant No. 30916014107, the U.S. National Science Foundation under Grant Nos. 1613950, 1115417, the Program for Changjiang Scholars and Innovative Research Team in University (PCSIRT) of the Ministry of Education, China, under Grant No. IRT13059.

\section{References}

[1] Cao F., Ye H., and Wang D. A probabilistic learning algorithm for robust modeling using neural networks with random weights. Information Sciences, 313, pp. 62-78, 2015.

[2] Cormack G. V. and Kolcz A. Spam filter evaluation with imprecise ground truth. In Proceedings of the 32nd International ACM SIGIR Conference on Research and Development in Information Retrieval (SIGIR), pp. 604-611, 2009.

[3] Costa-Jussà M. R., Grivolla J., Mellebeek B., Benavent F., Codina J., and Banchs R. E. Using annotations on Mechanical Turk to perform supervised polarity classification of Spanish customer comments. Information Sciences, 275, pp. 400-412, 2014.

[4] Dalvi N, Dasgupta A, Kumar R, and Rastogi V. Aggregating crowdsourced binary ratings. In Proceedings of the 22nd International Conference on World Wide Web, pp. 285-294, 2013.

[5] Dawid A. P. and Skene A. M. Maximum likelihood estimation of observer error-rates using the EM algorithm. Journal of Royal Statistical Society (Applied Statistics), 28(1), pp.20-28, 1979.

[6] Demartini G., Difallah D. E., and Cudré-Mauroux P. ZenCrowd: Leveraging probabilistic reasoning and crowdsourcing techniques for large-scale entity linking. In Proceedings of the $21 \mathrm{st}$ International Conference on World Wide Web, pp. 469-478, 2012.

[7] Díez-Pastor J. F., Rodríguez J. J., García-Osorio C. I., and Kuncheva L. I. Diversity techniques improve the performance of the best imbalance learning ensembles. Information Sciences, 325, pp. 98-117, 2015.

[8] Doan A., Ramakrishnan R., and Halevy A. Y. Crowdsourcing systems on the World Wide Web. Communications of the ACM, 54(4), pp. 86-96, 2011.

[9] Downs J. S., Holbrook M. B., Sheng S., and Cranor L. F. Are you participants gaming the system? Screen Mechanical Turk workers. In Proceedings of the 28th ACM SIGCHI Conference on Human Factors in Computing Systems, pp. 2399-2402, 2011.

[10] Grady C. and Lease M. Crowdsourcing document relevance assessment with Mechanical Turk. In Proceedings of the NAACL HLT 2010 Workshop on Creating Speech and Language Data with Amazons Mechanical Turk, pp. 172-179, 2010.

[11] Bin G. and Sheng V. S. A robust regularization path algorithm for $v$-support vector classification. 
IEEE Transactions on Neural Networks and Learning Systems, 2016. DOI: 10.1109/TNNLS.2016.2527796.

[12] Bin G., Sheng V. S., Wang Z., Ho D., Osman S., and Li S. Incremental learning for v-support vector regression. Neural Networks, 67, pp. 140-150, 2015.

[13] Bin G., Sun X., and Sheng V. S. Structural minimax probability machine. IEEE Transactions on Neural Networks and Learning Systems, 2016. DOI: 10.1109/TNNLS.2016.2544779.

[14] Howe J. Crowdsourcing: How the power of the crowd is driving the future of business. Random House, New York, 2008.

[15] Ipeirotis P. G., Provost F., Sheng V. S., and Wang J. Repeated labeling using multiple noisy labelers. Data Mining and Knowledge Discovery, 28(2), pp.402-441, 2014.

[16] Ipeirotis P. G., Provost F., and Wang J. Quality management on Amazon Mechanical Turk. In Proceedings of the ACM SIGKDD Workshop on Human Computation, pp. 64-67, 2010.

[17] Jung H. J. and Lease M. Improving consensus accuracy via z-score and weighted voting. In Proceedings of the 3rd Human Computation Workshop at AAAI, pp. 88-90, 2011.

[18] Kajino H., Tsuboi Y., and Kashima H. A Convex formulation for learning from crowds. Transactions of the Japanese Society for Artificial Intelligence, 27, pp. 133-142, 2012.

[19] Kara Y. E., Genc G., Aran O. and Akarun L. Modeling annotator behaviors for crowd labeling. Neurocomputing, 160, pp.141-156, 2015.

[20] Karger D. R., Oh S., and Shah D. Budget-optimal crowdsourcing using low-rank matrix approximations. In the 49th Annual Conference on Communication, Control, and Computing, pp. 284-291, 2011

[21] Klebanov B. B. and Beigman E. Some empirical evidence for annotation noise in a benchmarked dataset. In Human Language Technologies: The 2010 Annual Conference of the North American Chapter of the Association for Computational Linguistics, pp. 438-446, 2010.

[22] Kumar A. and M. Lease. Modeling annotator accuracies for supervised learning. In the 4th ACM WSDM Workshop on Crowdsourcing for Search and Data Mining, pp. 19-22, 2011.

[23] Kurve A., Miller D. J., and Kesidis G. Multicategory crowdsourcing accounting for variable task difficulty, worker skill, and worker intention. IEEE Transactions on Knowledge and Data Engineering, 27(3), pp.794-809, 2015.

[24] Liu K., Cheung W. K., and Liu J. Detecting multiple stochastic network motifs in network data. Knowledge and Information Systems, 42(1), pp. 49-74, 2015.

[25] Miller G. A. and Charles W. G. Contextual correlates of semantic similarity. Language and Cognitive Processes, 6(1), pp. 1-28, 1991.

[26] Muhammadi J., Rabiee H. R., and Hosseini A. A unified statistical framework for crowd labeling. Knowledge and Information Systems, 45(2), pp. 271-294, 2015.

[27] Ng S. K., Krishnan T., and McLachlan, G. J., 2012. The EM algorithm. In Handbook of computational statistics, Springer Berlin Heidelberg, pp. 139-172.

[28] Nguyen Q. V. H., Lam Ngoc T., Nguyen Thanh T., and Aberer K. An evaluation of aggregation techniques in crowdsourcing. In Proceedings of the 14th International Conference on Web Information System Engineering, pp. 1-15, 2013.

[29] Prati R. C., Batista G. E., and Silva D. F. Class imbalance revisited: a new experimental setup to assess the performance of treatment methods. Knowledge and Information Systems, 45(1), pp. 247-270, 2015. 
[30] Raykar V. C., Yu S., Zhao L. H., Florin C., Valadez G. H., Bogoni L., and Moy L. Learning from crowds. Journal of Machine Learning Research 11, pp.1297-1322, 2010.

[31] Ross J., Irani L., Silberman M. S., Zaldivar A., Tomlinson B. Who are the crowdworkers? Shifting demographics in Mechanical Turk. In Proceedings of the 28th ACM SIGCHI Conference on Human Factors in Computing Systems, pp. 2863-2872, 2010.

[32] Sheng V. S., Provost F., and Ipeirotis P. Get another label? Improving data quality and data mining using multiple, noisy labeler. In Proceedings of the ACM SIGKDD International Conference on Knowledge Discovery and Data Mining, pp. 614-662, 2008.

[33] Sheshadri A. and Lease M. SQUARE: A benchmark for research on computing crowd consensus. In Proceedings of the First AAAI Conference on Human Computation and Crowdsourcing, pp. 156-164, 2013.

[34] Smyth P., Burl M. C., Fayyad U. M., Perona P., and Baldi P. Inferring ground truth from subjective labeling of Venus images. In Proceedings of the Conference of Neural Information Process Systems, pp. 1085-1092, 1995.

[35] Snow R., O'Connor B., Jurafsky D., and Ng A. Cheap and fast-but is it good?: Evaluating non-expert annotations for natural language tasks. In Proceedings of the Conference on Empirical Methods in Natural Language Processing, pp. 254-263, 2008.

[36] Sorokin A. and Forsyth D. Utility data annotation with Amazon Mechanical Turk. In Computer Vision and Pattern Recognition Workshops, pp. 1-8, 2008.

[37] Strapparava C. and Mihalcea R. SemEval-2007 Task 14: Affective text. In Proceedings of the 4th International Workshop on the Semantic Evaluations, pp. 70-74, 2007.

[38] Su Q., Pavlov D., Chow J. H., and Baker W. C. Internet-scale collection of human-reviewed data. In Proceedings of the 16th International Conference on World Wide Web, pp. 231-240, 2007.

[39] Sun K., Li P., Tao W., and Tang Y. Feature guided biased Gaussian mixture model for image matching. Information Sciences, 295, pp. 323-336, 2015.

[40] Tang W. and Lease M. Semi-supervised consensus labeling for crowdsourcing. In SIGIR 2011 Workshop on Crowdsourcing for Information Retrieval, pp. 36-41, 2011.

[41] Welinder P., Branson S., Perona P., and Belongie S. J. The multidimensional wisdom of crowds. In Advances in Neural Information Processing Systems, pp. 2424-2432, 2010.

[42] Whitehill J., Ruvolo P., Wu T., Bergsma J., and Movellan J. Whose vote should count more: Optimal integration of labels from labelers of unknown expertise. In Advances in Neural Information Processing Systems, pp. 2035-2043, 2009.

[43] Zhang J., Wu X., and Sheng V. S. Imbalanced multiple noisy labeling. IEEE Transactions on Knowledge and Data Engineering, 27(2), pp. 489-503, 2015.

[44] Zhang J., Wu X., and Sheng V. S. Active Learning with Imbalanced Multiple Noisy Labeling. IEEE Transactions on Cybernetics 45(5), pp. 1081-1093, 2015.

[45] Zhu J., Xie Q., and Zheng K. An improved early detection method of type-2 diabetes mellitus using multiple classifier system. Information Sciences, 292, pp. 1-14, 2015.

[46] Zhuang $\mathrm{H}$ and Young J. Leveraging in-batch annotation bias for crowdsourced active learning. In Proceedings of the Eighth ACM International Conference on Web Search and Data Mining, pp. 243-252, 2015. 